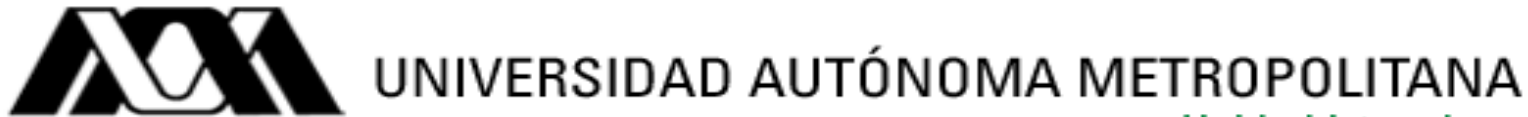

\section{Estimación del equilibrio hídrico tisular en hemodiálisis mediante espectroscopía de reactancia}

Comunicación de resultados para obtener grado de MAESTRA EN CIENCIAS (Ingeniería Biomédica) de

Ing. Ericka Stanford Alcántara

Enero 2014

Asesor: Dr. Emilio Sacristán Rock

Jurado Calificador:

Presidente: Dr. Héctor Alejandro Pérez Grovas Garza, INCICh

Vicepresidente: Dr. Emilio Sacristán Rock, UAM-I

Vocal: Cesar Antonio González, Escuela Militar de Graduados de Sanidad. 


\section{DU UNIVERSIDAD AUTÓNOMA METROPOLITANA Casa ablerta al tiempo

\section{Estimación del equilibrio hídrico tisular en hemodiálisis mediante espectroscopía de reactancia}

Comunicación de resultados para obtener grado de MAESTRA EN CIENCIAS (Ingeniería Biomédica) de

Ing. Ericka Stanford Alcántara

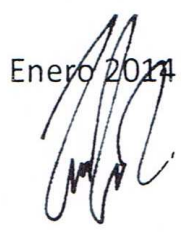

Asesor: Dr. Emilio Sacristán Rock

Jurado Calificador:

Presidente: Dr. Héctor Alejandro Pérez Grovas Garza, INCICh Vicepresidente: Dr. Emilio Sacristán Rock, UAM-I Vocal: Cesar Antonio González, Escuela Militar de Graduados de Sanidad.

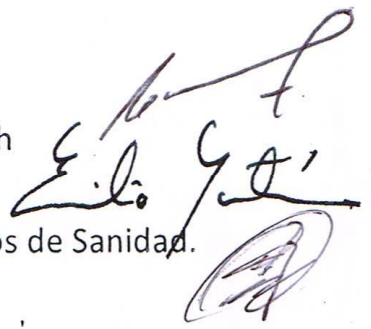




\section{Agradecimientos}

Agradezco al Instituto Nacional de Cardiología "Ignacio Chávez", por permitirme realizar este proyecto dentro de sus instalaciones; en especial al Dr. Héctor Pérez Grovas y al personal médico de la unidad de hemodiálisis por todo el apoyo y la ayuda que me brindaron durante mi estancia. En segundo lugar me gustaría agradecer al Consejo Nacional de Ciencia y Tecnología (CONACYT), Universidad Autónoma Metropolitana (UAM-I) y al Centro Nacional de Investigación en Instrumentación e Imagenología Médica $\left(\mathrm{Cl}^{3} \mathrm{M}\right)$ por su apoyo y los recursos brindados para el desarrollo de este proyecto de investigación y mis estudios de maestría.

De manera especial agradezco a mi asesor el Dr. Emilio Sacristán Rock por todas sus enseñanzas y su guía en durante todas las etapas de este proyecto. Así como a la Dra. Nohra Elsy Beltran Vargas por facilitarme el software para la obtención de los modelos matemáticos, y orientarme en el uso del mismo. Por último le agradezco a mis compañeros y amigos: Ing. Rafael Hernández y al Ing. Francisco Flores por todo su apoyo y consejos durante mi formación. 


\section{Índice}

Agradecimientos____ i

Índice___ ii

Abreviaturas__iv

Resumen___ v

1. Introducción 1

2. Antecedentes

2.1. Insuficiencia renal crónica y hemodiálisis___ 5

2.2. Equilibrio hídrico___ 6

2.3. Equilibrio hídrico en hemodiálisis _ 7

2.4. Técnicas para la estimación del estado hídrico en $\mathrm{HD}$

2.5. Impedancia eléctrica____ 10

2.5.1. Actividad eléctrica del tejido vivo___ 11

2.5.2. Regiones de dispersión de los tejidos biológicos____ 12

2.5.3. Análisis de bioimpedancia___ 13

2.6. Bioimpedancia y sus clasificaciones___ 15

2.7. Bioimpedancia en hemodiálisis___ 16

2.8. Espectrómetro de impedancia___ 19

3. Materiales y Métodos__ 21

3.1. Ubicación de electrodos___ 21

3.2. Electrodos de Ag-AgCl___ 21

3.3. Geometría fija entre electrodos___ 22

3.4. Población en hemodiafiltración___ 22

3.4.1. Criterios de inclusión___ 22

3.4.2. Criterios de exclusión___ 23

3.4.3. Procedimiento___ 23

3.4.4. Instrumentación__ 24

3.4.5. Análisis de datos__ 24

3.5. Población de voluntarios sanos___ 24

3.5.1. Criterios de inclusión___ 24

3.5.2. Criterios de exclusión___ 25

3.5.3. Criterios de rechazo__ 25

3.5.4. Procedimiento _ 25

3.5.5. Instrumentación _ 25

3.5.6. Análisis de datos__ 25 
4. Resultados

4.1. Descripción de la población 27

4.2. Espectros típicos. 28

4.3. Comportamiento de reactancia en HDF__ 30

4.4. Reactancia central durante HDF___ 31

4.5. Reactancia en sujetos sanos___ 32

4.6. Comparación de Xh de ambas poblaciones___ 34

4.7. Cambio de reactancia central y de volumen ultrafiltrado.___ 34

4.8. Valor predictivo de la reactancia___ 37

5. Discusión

6. Conclusiones y recomendaciones

6.1. Conclusiones 43

6.2. Trabajo futuro 44

Referencias 45 


\section{Lista de abreviaturas}

\begin{tabular}{ll} 
ACT & Agua corporal total \\
BIA & Análisis de bioimpedancia \\
BIAV & Análisis de bioimpedancia vectorial \\
EBI & Espectroscopía de bioimpedancia \\
HD & Hemodiálisis \\
HDF & Hemodiafiltración \\
IRC & Insuficiencia renal crónica \\
LEC & Líquido extracelular \\
LIC & Líquido intracelular \\
P_máx VUF & Pendiente máxima del cambio en el volumen de ultrafiltrado \\
P_máx Xh & Pendiente máxima del cambio en reactancia central \\
R & Resistencia \\
Rh & Resistencia central para altas frecuencias \\
VEC & Volumen extracelular \\
VIC & Volumen intracelular \\
X & Reactancia \\
Xh & Reactancia central para altas frecuencias \\
\hline
\end{tabular}




\section{Resumen}

El equilibrio hídrico tisular es el balance de agua y electrolitos que existe entre los compartimentos intracelular y extracelular; durante las sesiones de hemodiálisis (HD) se extrae líquido del espacio extracelular afectando el equilibrio hídrico del paciente; el cual puede ser medido a través la espectroscopía de bioimpedancia (EBI). Esta técnica relaciona el volumen extracelular e intracelular mediante la reactancia. En la literatura existen varios estudios comparativos entre bioimpedancia de cuerpo completo y el análisis segmentado, donde se encontró que el $90 \%$ de los cambios de impedancia se deben a las piernas y brazos [3].Sin embargo, las piernas están más expuestas a la acumulación de líquidos por efectos gravitatorios y a artefactos de movimiento debidos a cambios de posición, ejercicio y movimientos intrínsecos del paciente; por lo que los brazos son una mejor zona para realizar los estudios durante HD.

Aunado a esto, los electrodos y los cables de registro actúan como antenas que contribuyen a la baja reproducibilidad de las mediciones a demás de que existe una gran variación en la colocación de los electrodos y por lo tanto la región transversal de tejido a estudiar. La geometría fija de los electrodos promete resolver este problema ya que no permite variaciones entre la distancia de los electrodos y la región transversal, además de que disminuye la distancia entre electrodos reduciendo el efecto antena de los cables de registro. De ahí que para mejorar la reproducibilidad de las mediciones se deben minimizar los artefactos de movimiento y optimizar la colocación de

los electrodos. Por ello, este proyecto evaluó mediante EBI del antebrazo el equilibrio hídrico tisular de los pacientes durante la sesión de hemodiafiltración.

Se realizaron estudios de EBI en dos poblaciones: 1 ) sujetos sanos ( 3 hombres y 1 mujer) y 2) pacientes en hemodiafiltración ( 3 mujeres y 1 hombre). La población sana fue analizada en: ayuno, ante la ingesta de $1 \mathrm{~L}$ de agua y 1 hora después de la ingesta. Se encontraron diferencias estadísticamente significativas ( $p=0.035$ ) en la reactancia en ayuno y 1 hora después de la ingesta; Lo que permitió validar el uso de la técnica en el antebrazo. Mientras que para la población en hemodiafiltración se realizaron mediciones durante toda la sesión cada 15 min para tres sesiones diferentes. Se encontraron diferencias significativas $(p=0.0085)$ en el cambio de la reactancia en el tiempo. Los resultados del análisis de curvas ROC sugieren que el monitoreo de la reactancia durante las sesiones de hemodiafiltración permitirá controlar la dosis de diálisis evitando el evento adverso (hipotensión, mareo, vómito, calambres,etc.) debido al desequilibrio hídrico. 


\section{Introducción}

En los pacientes con insuficiencia renal crónica (IRC) la capacidad de excretar agua en orina se ve afectada reduciendo el volumen urinario diario. En dichos pacientes es notoria la incapacidad de mantener un balance entre el agua ingerida y el agua excretada. El resultado es la retención de agua que clínicamente se aprecia con aumento de peso y edema. La hemodiálisis (HD) es uno de los tratamientos renales sustitutivos existentes, el cual permite remover las toxinas y el exceso de líquidos y electrólitos del cuerpo mediante el uso de una máquina y un dializador, también conocidos como riñón artificial.

Sin embargo, aún no se tiene correctamente cuantificada la cantidad de agua que tiene que ser removida del paciente. Existe una aproximación del peso del paciente si no existiera un exceso de agua el cual como su nombre lo indica es el peso seco y en base a la diferencia del peso del paciente antes de la sesión y el peso seco se determina la cantidad de agua que se deberá extraer, no obstante, llegar al peso seco no necesariamente significa alcanzar el equilibrio hídrico. Aunado a esto, el peso seco se modifica a través del tiempo como consecuencia del cambio en la composición corporal del paciente [1].Por consiguiente, el peso seco no debería de ser el indicador del volumen de extracción de líquido del paciente, ya que no representa apropiadamente el estado hídrico que el paciente deberá conseguir al finalizar el tratamiento [2].

A diferencia del peso seco el balance hídrico no es un número al cual se debe de llegar al final de la sesión, más bien el equilibrio hídrico es una variable a controlar durante todo el tratamiento. El estado de hidratación del paciente durante las sesiones de HD es de vital importancia, ya que existen múltiples complicaciones que derivan del desequilibrio hídrico; una de las principales es la hipotensión, la cual ocurre entre el $20-50 \%$ de las sesiones, a pesar de que esta complicación es multifactorial entre sus posibles causas se encuentra la deshidratación, es decir, una remoción excesiva de líquido. Por el contrario, si no se extrae completamente el excedente de agua el cuerpo intenta compensar el exceso de volumen aumentando la presión arterial. Si el paciente es sometido constantemente a un estado hipertensivo, la carga excesiva de trabajo para el corazón puede llevar al desarrollo de insuficiencia cardíaca y cardiopatía isquémica. Otras de las posibles complicaciones durante hemodiálisis son: cefaleas, vómitos, calambres, nauseas y malestar general [3].

El equilibrio hídrico no es más que el balance de agua que existe entre los diferentes compartimentos del cuerpo, el cuerpo humano se divide principalmente en dos compartimentos el intracelular y el extracelular, el volumen de agua y la concentración de electrolitos presentes en ambos compartimentos es lo que determina el equilibrio hídrico; no obstante, la pregunta sigue siendo: ¿Cómo medirlo? En la actualidad existen diferentes técnicas para identificar el estado de hidratación: Radiografía de tórax, ecografía de vena cava, biomarcadores cardíacos, valoración del volumen plasmático, bioimpedancia eléctrica, etc. Dentro de las técnicas más utilizadas todas a excepción de la bioimpedancia eléctrica presentan algún inconveniente, ya sean riesgos asociados, el costo o la dificultad para llevar a cabo la medición. Por lo que la bioimpedancia eléctrica resulta 


\section{Ericka Stanford Alcántara}

ser el método más utilizado en la clínica debido a que es fácil de usar, no costosa y no invasiva (ver tabla 1) [4].

La aplicación del análisis de bioimpedancia eléctrica ha permitido conocer no solo el estado de hidratación del paciente, sino también su composición corporal (masa magra, masa grasa, etc.), lo cual aplicado a hemodiálisis ha permitiendo realizar modificaciones en la dosis de diálisis para poder efectuar un mejor tratamiento. A pesar de estos avances, la aplicación de bioimpedancia en hemodiálisis aún se está analizando ya que existen discrepancias entre la comunidad médica en cuanto a la forma de realizar la medición. Se han realizado varios estudios comparativos entre bioimpedancia de cuerpo completo y el análisis segmentado, donde se encontró que las mediciones de impedancia que se realizan en las extremidades están más relacionadas a los cambios hídricos del paciente ya que el $90 \%$ de los cambios en impedancia se deben a los cambios hídricos de piernas y brazos [3].

El actual estado de la técnica ha permitido consolidar la bioimpedancia como un método eficaz para determinar la cantidad de líquido en el cuerpo, no obstante la aplicación de esta técnica en hemodiálisis sigue siendo utilizada como método para evaluar la composición corporal del paciente más que para determinar el balance hídrico, debido a que la mayoría de los estudios requieren que el paciente adopte una posición estática para poder realizar la medición. Sí bien la composición corporal resulta ser información importante para el seguimiento del paciente, la monitorización del equilibrio hídrico a lo largo del tratamiento es fundamental para regular la dosis (velocidad y cantidad del ultrafiltrado) y poder realizar tratamientos de mayor calidad que reduzcan las complicaciones asociadas a la hemodiálisis.

Por ello, este proyecto pretender evaluar mediante espectroscopia de bioimpedancia (EBI) del antebrazo, el equilibrio hídrico tisular de los pacientes durante la sesión de hemodiafiltración.

\section{Hipótesis}

Los cambios en el equilibrio hídrico de los pacientes en hemodiafiltración están relacionados con los cambios en reactancia, por lo que es posible medir el estado hídrico del sujeto mediante espectroscopia de reactancia.

\section{Objetivo General}

Desarrollar un sistema de medición del equilibrio hídrico tisular del paciente hemodialítico mediante espectroscopia de bioimpedancia capaz de realizar mediciones reproducibles intra e interpaciente durante todo el tratamiento. 


\section{Objetivos Específicos}

- Diseñar un arreglo de electrodos de superficie con geometría constante para el antebrazo.

- Validación del dispositivo mediante experimentación en una población de pacientes en tratamiento de hemodiafiltración.

- Evaluar los cambios entre la reactancia y el volumen ultrafiltrado.

- Evaluar el monitoreo de la reactancia como método para controlar la tasa de diálisis. 
Estimación del equilibrio hídrico tisular en hemodiálisis mediante espectroscopía de reactancia Ericka Stanford Alcántara 


\section{Antecedentes}

\subsection{Insuficiencia renal crónica y hemodiálisis}

La insuficiencia renal crónica (IRC) es un padecimiento irreversible en los riñones que va deteriorando su capacidad para depurar las toxinas de la sangre. La función renal se expresa por medio del filtrado glomerular (FG) o por el aclaramiento de creatinina $<60 \mathrm{ml} / \mathrm{min} / 1,73 \mathrm{~m} 2$, o bien como daño renal persistente de por lo menos 3 meses [5]. La manifestación más grave de la IRC es la insuficiencia renal crónica terminal (IRCT) o IRC estadio 5 la cual requiere de tratamiento sustitutivo mediante diálisis o trasplante renal.

La hemodiálisis es un tratamiento sustitutivo de la función renal que busca eliminar el exceso de toxinas, electrólitos y líquidos de la sangre. En este proceso la sangre se extrae del paciente hacia un circuito cerrado extracorpóreo mediante una bomba peristáltica y se hace pasar a través de un filtro también llamado dializador. El dializador se conforma por una serie de microtúbulos los cuales actúan como membranas semipermeables que separan la sangre del líquido de diálisis.

La remoción de toxinas se lleva a cabo mediante dos procesos simultáneos: 1) difusión y 2) convección. La difusión consta del movimiento de moléculas del lugar de mayor concentración al de menor concentración, es decir, de la sangre hacia el líquido de diálisis, sin embargo, las membranas semipermeables solo permiten el paso a moléculas de cierto tamaño lo que evita que moléculas grandes como las proteínas sanguíneas salgan de la sangre. El otro proceso que regula el intercambio de toxinas y agua es mediante convección, este se lleva a cabo cuando existe un gradiente de presión entre dos líquidos separados por una membrana, gracias a la diferencia de presión las moléculas de agua salen de la sangre hacia el líquido de diálisis que se encuentra a menor presión. Cabe destacar, que el movimiento de convección arrastra consigo algunas toxinas que son capaces de traspasar la membrana. Una vez que la sangre sale del dializador regresa al paciente a través del circuito extracorpóreo como se muestra en la figura 1. Las sesiones de HD se efectúan de forma periódica 2-3 veces a la semana. Cada sesión tiene una duración aproximada de $4 \mathrm{hrs}$, tiempo en el cual se "elimina" el exceso de agua y toxinas de la sangre.

En la búsqueda por mejorar el tratamiento se han desarrollado diferentes versiones de hemodiálisis, la hemofiltración (HF) y la hemodiafiltración (HDF). La HF utiliza el mismo principio que la HD con la diferencia de que el filtro es más poroso provocando que más solutos sean capaces de atravesar la membrana, otra diferencia es que al ultrafiltrar la sangre es necesario reponer el volumen de agua y electrolitos perdido agregando solución salina a la sangre postfiltración.

Por el otro lado la HDF resulta ser la combinación de la HD y la HF. Por lo que es posible eliminar toxinas de bajo y alto peso molecular, al igual que la $\mathrm{HF}$ es necesario reponer líquido y electrolitos post-filtrado. Actualmente la HDF ha sido la variante que ha otorgado un mejor control hemodinámico del paciente, reduciendo así el evento adverso. 


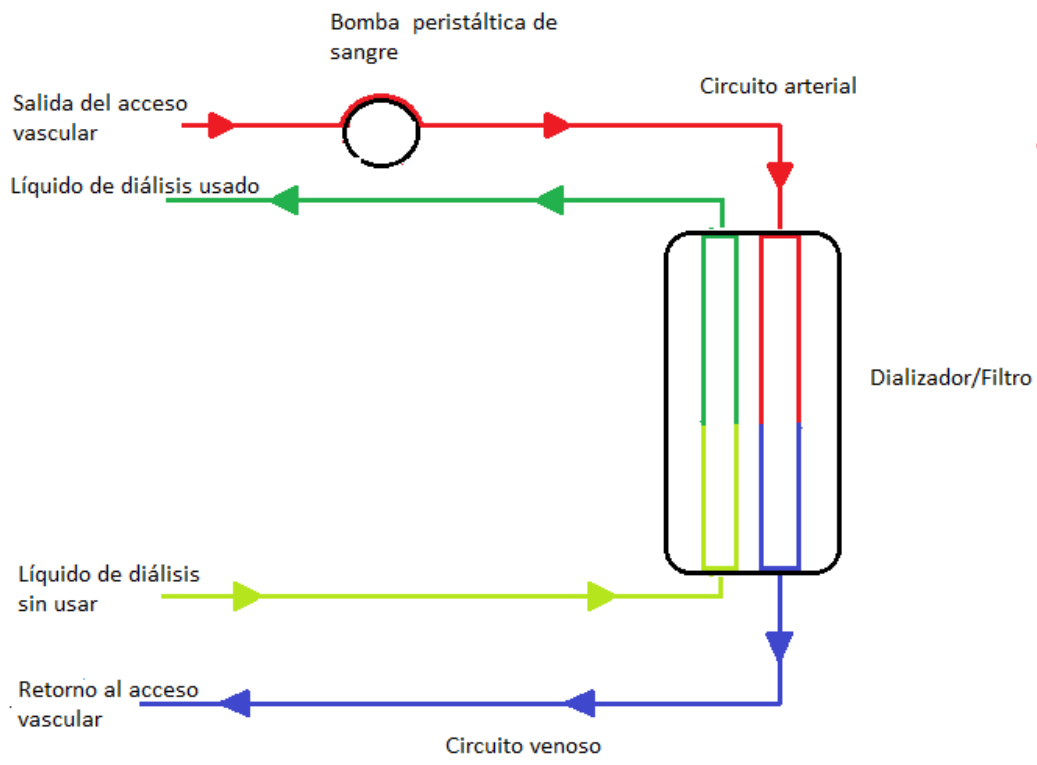

Figura 1. Esquema de hemodiálisis

\subsection{Equilibrio hídrico}

En el cuerpo humano debe de existir un balance entre la cantidad de líquidos que entran y salen del cuerpo para mantener la homeostasis. Por ello el volumen de líquidos ingeridos a través del agua y los alimentos son eliminados mediante el sudor, las heces y la orina. De tal forma que la cantidad de líquidos presentes en el cuerpo es relativamente constante en el transcurso del día. La importancia de balancear correctamente los líquidos radica en que aproximadamente el $60 \%$ del peso corporal total de un adulto promedio es agua. Toda esta agua se encuentra distribuida en el cuerpo y la podemos clasificar en dos compartimentos principales: a) el líquido extracelular (LEC) y b) el líquido intracelular (LIC). EI LEC a su vez se divide en líquido intersticial y plasma sanguíneo los cuales representan aproximadamente el $20 \%$ del peso de una persona promedio como se muestra en la figura 2. Mientras que el líquido intracelular, es decir, el líquido que se encuentra al interior de las más de 75 billones de células representa el $40 \%$ del peso corporal [6].

En promedio una persona sana ingiere $2300 \mathrm{ml}$ de agua a través de alimentos y bebidas mismos que son eliminados en el transcurso del día, mientras que en hemodiálisis se extraen en promedio 4 litros en 3-4 horas lo que hace bastante agresivo a este tratamiento por el hecho de remover un gran volumen de agua en tan poco tiempo, por lo mismo los pacientes se ven sometidos a cambios drásticos en el estado de hidratación, logrando pasar de la sobrehidratación a la deshidratación en cuestión de horas. 


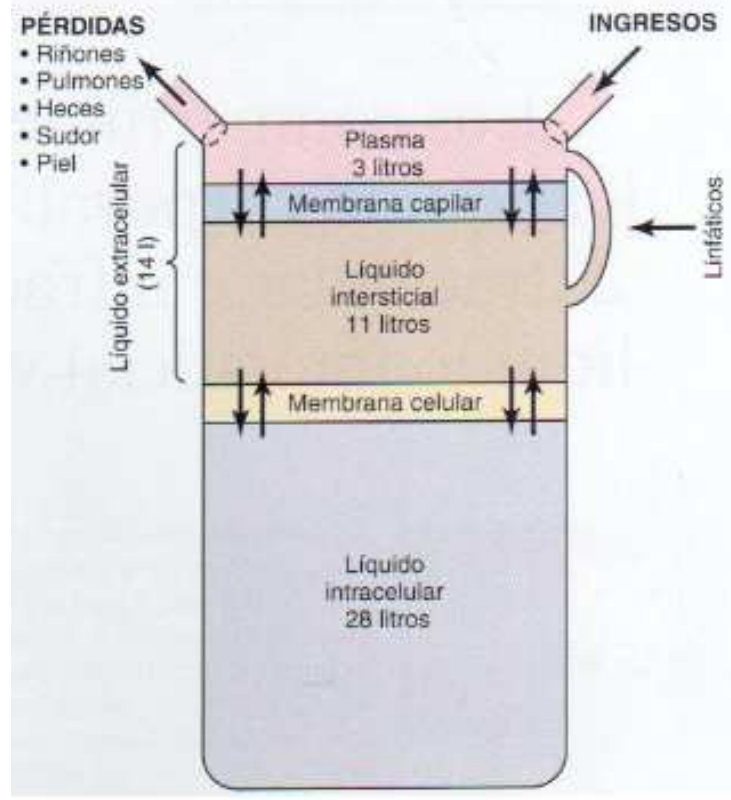

Figura 2.Distribución de líquidos corporales en sus diferentes compartimentos [6].

En HD lo más común es que los pacientes presenten una sobrehidratación importante antes de recibir el tratamiento, al exceso de líquido corporal se le conoce como edema y puede ser intra o extracelular; siendo más común el edema extracelular el cual ocurre generalmente debido a dos causas: 1) fuga del líquido del plasma al espacio intersticial mediante los capilares y 2) la incapacidad del sistema linfático de devolver líquido del intersticio al plasma. También existen varios trastornos que provocan edema extracelular: Insuficiencia renal crónica, insuficiencia cardiaca, obstrucción venosa, quemaduras, malnutrición proteica, etc. En particular el edema por IRC se debe a la disminución en la depuración urinaria provocando que el exceso de agua y cloruro de sodio permanezcan en el espacio extracelular y en consecuencia se produce un estado hipertensivo [6].

\subsection{Equilibrio hídrico en hemodiálisis.}

Como se explicó anteriormente, durante el tratamiento de hemodiálisis se extraen aproximadamente de 1 a 4 litros de agua mediante el filtrado de la sangre, provocando un movimiento constante de líquidos corporales de un compartimento a otro [7]. Cabe recordar que los tres compartimentos plasma, intersticio e intracelular guardan una relación de equilibrio osmótico y de presión con el compartimento vecino. De tal manera que el plasma esta en equilibrio con el intersticio y el intersticio a su vez esta en equilibrio con el líquido intracelular. Al extraer líquido desde el plasma se desequilibran los dos compartimentos (plasma e intersticio) provocando que el líquido del intersticio fluya hacia el plasma para re-equilibrar los gradientes osmóticos y de presión hasta alcanzar un nuevo punto de equilibrio, este proceso ocurre durante todo el tratamiento de hemodiálisis; Sin embargo si se extrae demasiado líquido del plasma el volumen del intersticio se verá disminuido ocasionando que el equilibrio entre intersticio y el 


\section{Ericka Stanford Alcántara}

líquido intracelular se pierda provocando que el líquido intracelular fluya hacia el intersticio para re equilibrar los gradientes. Por lo que de manera general se espera que el volumen intersticial e intracelular del paciente en hemodiálisis después del tratamiento se vean disminuidos mientras que el volumen vascular se mantenga más o menos constante como se muestra en la figura 3.
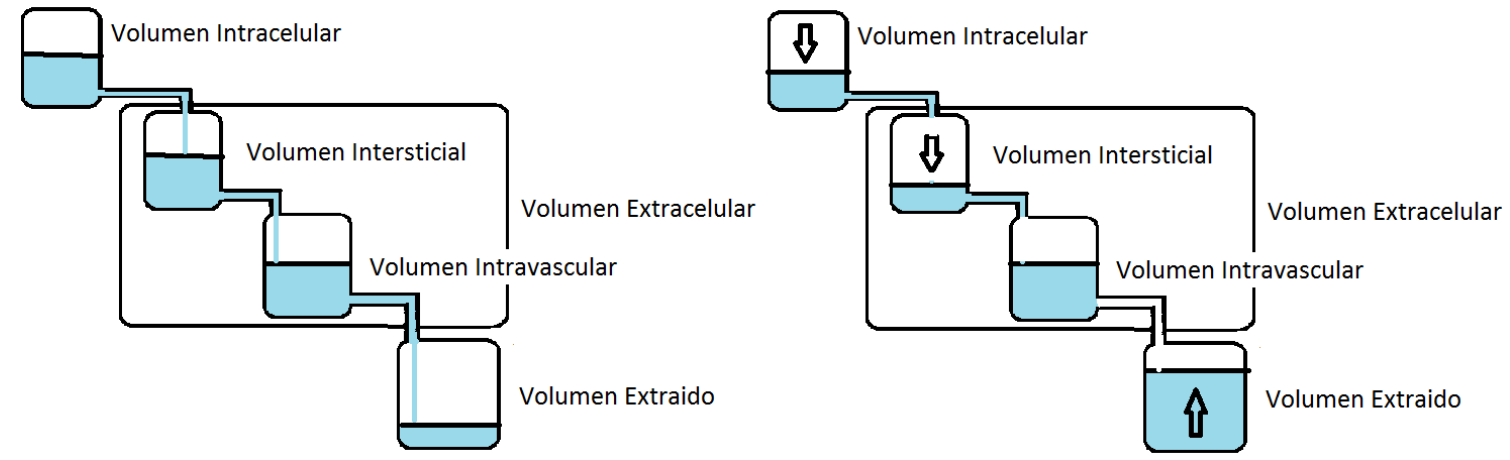

Figura 3. En la imagen de la izquierda se ilustra el movimiento de líquido durante hemodiálisis, mientras que la imagen de la derecha muestra el nuevo equilibrio hídrico una vez finalizado el tratamiento.

Los factores en HD que afectan el equilibrio hídrico son la cantidad de líquido extraída o que la velocidad de extracción sea muy rápida, ambas situaciones impiden que los mecanismos de regulación mantengan el equilibrio hídrico lo que se traduce en la descompensación del paciente, de ahí la importancia de conocer el balance hídrico del paciente.

\subsection{Técnicas para la estimación del estado hídrico en HD.}

Hoy por hoy no existe una herramienta que determine el equilibrio hídrico de manera adecuada, el actual estado de la técnica consiste en estimar el peso seco, el cual como su nombre lo indica es el peso que el paciente debería de tener sí no existiera un exceso de agua. Pese a que su estimación aún es intuitiva y está basada en una especie de ensayo y error sigue siendo el método utilizado por excelencia. La obtención del peso seco consisten en la evaluación de algunos parámetros clínicos como: la presencia de hipertensión o hipotensión intra e inter diálisis, la presencia de algún evento adverso y la ganancia de peso entre sesiones. Luego entonces resulta necesario implementar técnicas que evalúen correctamente el estado hídrico del paciente y dejar de lado las estimaciones intuitivas que se realizan hoy día.

Existen varias herramientas que pueden facilitar la determinación de estado de hidratación, como se muestra en la tabla 1: 


\section{Tabla 1.Técnicasmás utilizadas para estimar el estado de hidratación en HD.}

\begin{tabular}{|c|c|c|c|c|}
\hline & Técnica & Como funciona & Ventajas & Desventajas \\
\hline a) & $\begin{array}{l}\text { Dilución de } \\
\text { indicadores }\end{array}$ & $\begin{array}{l}\text { - Se determina el agua corporal total } \\
\text { (ACT) al inyectar agua pesada o agua } \\
\text { radioactiva, por otro lado el LEC se } \\
\text { determina al inyectar sustancias que no }\end{array}$ & $\begin{array}{l}\text { - Medición directa del } \\
\text { estado de hidratación } \\
\text { - Mediciones exactas } \\
\text { teóricamente. }\end{array}$ & $\begin{array}{l}\text { - Se requiere personal médico } \\
\text { capacitado. } \\
\text { - Los indicadores son costosos. }\end{array}$ \\
\hline
\end{tabular}
atraviesen la membrana ( $\mathrm{Na}, \mathrm{Cl}$ radioactivo). EI LIC se obtiene a partir de ACT-LEC.

Para determinar el volumen plasmático se inyecta algún colorante o Albúmina marcada con yodo radioactivo [6].

- Mide el volumen plasmático a través de su relación con el diámetro de la vena

b) Ecografía de cava inferior [2]. vena cava
- No invasiva

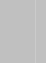


La mayoría de estas técnicas resultan complicadas de utilizar, costosas o invasivas lo que dificulta su uso rutinario como método de estimación del equilibrio hídrico. No obstante, existe una gran tendencia en los últimos 20 años hacia el uso de bioimpedancia debido a que es la única técnica hasta el momento que resulta fácil de usar, no invasiva y de bajo costo, aunque su precisión no se compara contra el estándar de oro; recientes investigaciones han mostrado que el método de espectroscopia de bioimpedancia puede ser tan preciso como los métodos de dilución tradicionales [8].

\subsection{Impedancia eléctrica}

La impedancia eléctrica es una propiedad inherente a los tejidos biológicos la cual sirve para caracterizarlos de manera no invasiva. Las primeras mediciones de bioimpedancia reportadas por Hoffer en 1969 correlacionaban los cambios en el agua corporal total y los cambios en impedancia, desde entonces la bioimpedancia ha sido tema de estudio para determinar la cantidad de agua en el espacio extracelular, intracelular, agua corporal total, masa magra, etc. [9]

El principio básico de medición de impedancia en un sistema geométrico que depende de la longitud, la forma del segmento y el área transversal. A pesar de que el cuerpo humano no es un conductor homogéneo se le puede considerar como un cilindro conductor de longitud igual a la talla del individuo y de sección transversal conocida (A), por el cual fluye una corriente alterna y donde se tiene una diferencia de potencial entre sus extremos, ver figura 4. De ahí la impedancia del cilindro depende de la resistividad del tejido ( $\rho$ ), la longitud (L) y la sección transversal (A), ver ecuación 1. A partir de esta ecuación se puede obtener el volumen del cilindro multiplicando el cociente de la ecuación 1 por $L / L$, partiendo del hecho de que $V=A / L$, de ahí se llega a la ecuación 2. [10]

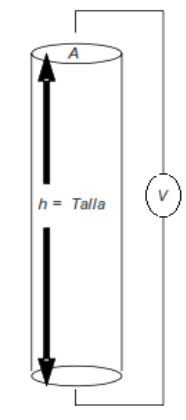

Figura 4. Modelo cilíndrico del cuerpo humano [7].

$$
\begin{aligned}
& Z=(\rho \times L) / A \\
& V=\left(\rho \times L^{z}\right) / Z
\end{aligned}
$$


De la ec.2 se puede estimar el volumen de agua dependiendo del valor de resistividad que se tenga. De esta forma la impedancia y el agua corporal total (ACT o TBW) se pueden obtener en términos de la talla del sujeto y la impedancia medida ec.3. [10]

$$
\mathrm{ACT}=\mathrm{L}^{2} / \mathrm{Z} \quad \text { (ec.3) }
$$

No obstante la geometría del cuerpo humano resulta más compleja que la de un cilindro homogéneo, por ello se han propuesto otros modelos geométricos que intentan asemejar de mejor manera la geometría humana, el principal modelo es el de 5 cilindros. El cual como su nombre lo indica consiste en un conjunto de 5 cilindros conectados entre sí como se muestra en la figura 5, los cuales corresponden a brazos, piernas y torso [10]. En la actualidad existe un modelo de 11 cilindros que corresponde a torso, brazos, antebrazos, muslos, rodillas y pantorrillas [11].

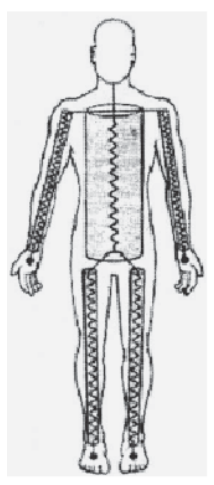

(a)

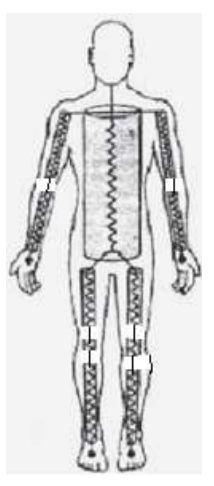

(b)

Figura 5. (a) Modelo de cinco cilindros del cuerpo humano y (b) Modelo de 11 cilindros [10,11].

Para los modelos geométricos se han estimado diferentes valores de impedancia para cada cilindro y el porcentaje que representa cada cilindro de la resistencia corporal total. Aunque los valores encontrados varían entre autores $[11,12]$ en general coinciden en que a el torso es el cilindro más grande y el que menos contribuye a la resistencia corporal total con tan solo $5.9 \%$ aprox. Esto se debe a la presencia de los diferentes órganos en la cavidad abdominal. Mientras que las zonas que más contribuyen a la resistencia total son las pantorrillas y los antebrazos con $30 \%$ y $25.3 \%$ respectivamente [11].

\subsubsection{Actividad eléctrica de tejido vivo}

La unidad fundamental de los tejidos es la célula, la cual básicamente es un contenedor de electrólitos y estructuras celulares. La célula se encuentra envuelta por la membrana celular compuesta por una bicapa lipídica formada por una parte hidrofílica y otra hidrofóbica la cual crea una barrera semipermeable que separa el interior y el exterior de la célula, ver figura 6. Gracias a las propiedades pasivas de la célula (canales iónicos, bomba $\mathrm{Na}-\mathrm{K}$, etc.) no todas las moléculas son capaces de a travesar la membrana celular, o bien, su transporte hacia y desde la célula depende 
de la disponibilidad de los canales iónicos. La diferencia de concentración de solutos entre ambos compartimentos celulares genera gradientes eléctricos, de concentración y de presión [13].

Las propiedades celulares pueden representarse con un circuito RC en paralelo nombrado así por tener una resistencia y un capacitor conectados en paralelo; el cual reproduce el comportamiento eléctrico de la célula como se ilustra en la figura 7. Donde, la resistencia eléctrica corresponde a la resistencia de los canales iónicos al flujo de iones y la membrana celular junto con los líquidos intra y extracelular forman un capacitor al ser dos placas conductoras (LIC y LEC) separados por un dieléctrico (membrana).

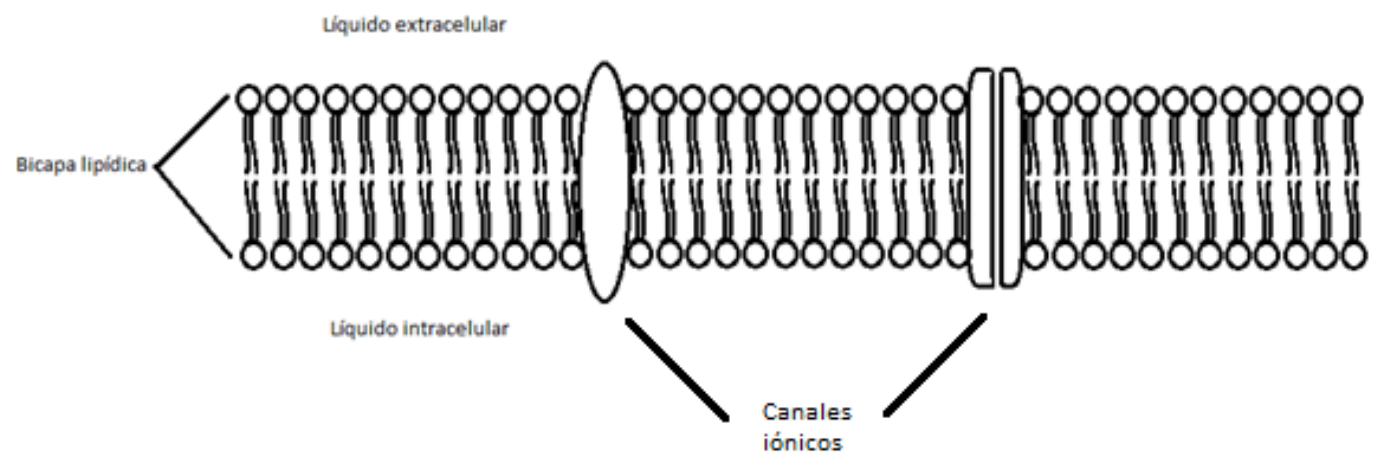

Figura 6. Membrana celular
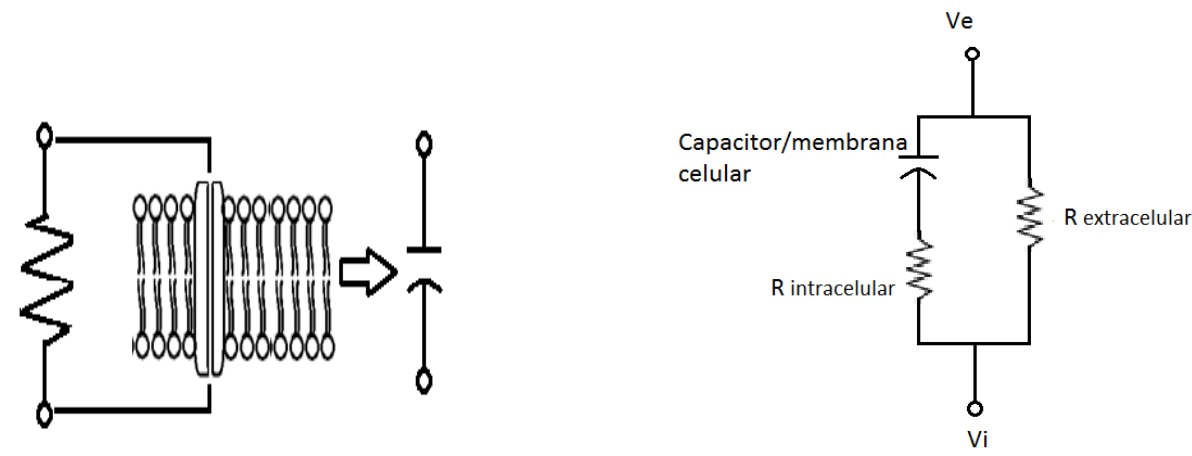

Figura 7. Circuito eléctrico equivalente de la célula.

\subsubsection{Regiones de dispersión de los tejidos biológicos.}

Dadas la propiedades eléctricas de los tejidos su impedancia cambia dependiendo de la frecuencia de excitación. Al realizar un barrido de frecuencias se observa que existen cambios abruptos en impedancia en rangos pequeños de frecuencias, a estas zonas se les llama región de dispersión. Para los tejidos biológicos se han identificado tres regiones de relajación o dispersión en rangos de frecuencia particulares: 
Alpha $(\boldsymbol{\alpha})$ : Es la primera región de dispersión y corresponde a las bajas frecuencias, desde unos cuantos hertz hasta $1 \mathrm{kHz}$ y se debe a la polarización de un solo lado de la membrana celular (extracelular) debido a la difusión de iones.

Beta $(\boldsymbol{\beta})$ : A esta región corresponden frecuencias de $10 \mathrm{kHz}$ hasta $10 \mathrm{MHz}$. En esta región se debe a la polarización de la membrana celular.

Gamma ( $\gamma$ ): Región de dispersión para altas frecuencias que corresponden de $100 \mathrm{MHz}$ en adelante y se debe principalmente al contenido de proteínas, agua y electrolitos de los tejidos.

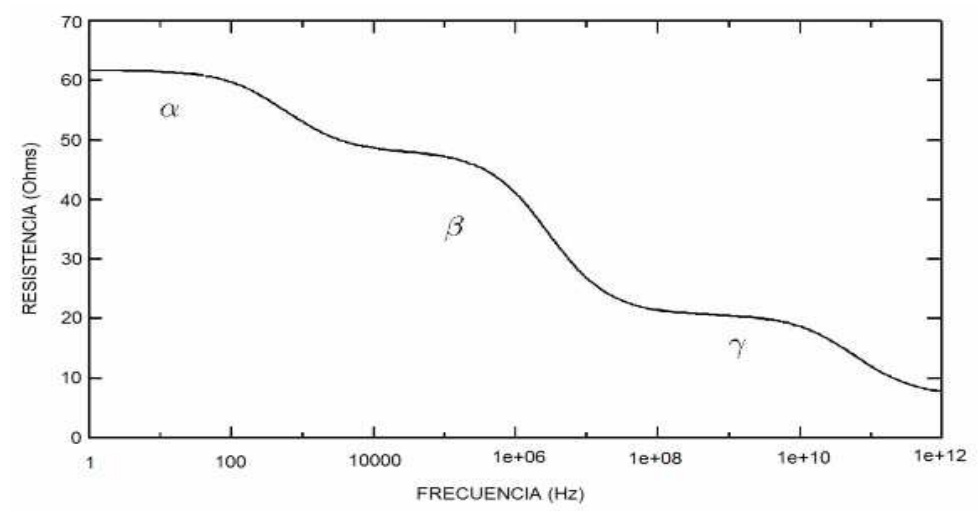

Figura 8. Regiones de dispersión de los tejidos biológicos [9].

\subsubsection{Análisis de bioimpedancia}

La impedancia desde el punto de vista matemático es un número complejo que puede representarse como la suma de un número real y un número imaginario, la parte real corresponde a la resistencia y la imaginaria a la reactancia recordando que la resistencia se relaciona al componente resistivo del circuito eléctrico y la reactancia corresponde al capacitor. Por ser un número complejo la impedancia puede ser expresada en magnitud y fase, donde la magnitud corresponde al tamaño del vector formado desde el origen al graficar la resistencia en el eje de los reales (Re) y reactancia en el eje de los imaginarios $(\mathrm{Im})$, mientras que la fase es el ángulo que se forma entre el eje horizontal y el vector de impedancia, este comportamiento se muestra en las ecuaciones 4 y 5 respectivamente.

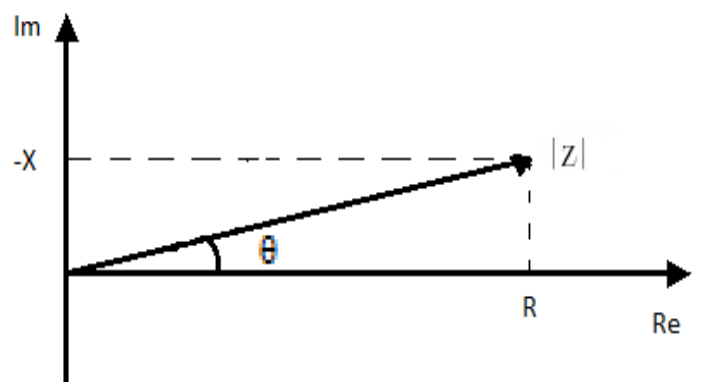

$$
|Z|=\sqrt{R^{2}+X^{2}} \quad \emptyset=\arctan \frac{R}{X}
$$

(ec.4) y (ec.5) 
Donde,

Z es la impedancia.

$R$ es la resistencia.

$X$ es la reactancia.

$\emptyset$ es el ángulo de fase.

En la figura anterior se observa que la reactancia esta expresada con valor negativo pero en el cuadrante que corresponde números imaginarios positivos, esto se debe a que la reactancia en sí es un valor negativo, sin embargo por convención se ha optado por graficarla de forma positiva para facilitar su interpretación clínica, por lo que no debe de resultar extraño encontrar que en la literatura la reactancia sea expresada de manera positiva o negativa, ambas formas de expresar la reactancia son correctas mientras que no se pierda de vista que la reactancia siempre corresponde a valores negativos.

El análisis de bioimpedancia en sí, mide la magnitud de la impedancia y el ángulo de fase; donde la impedancia " $Z$ " medida corresponde a la respuesta de los tejidos biológicos al paso de una corriente eléctrica a diferentes frecuencias [5]. Al inyectar una corriente eléctrica de baja frecuencia la corriente solo fluye a través del LEC ya que el capacitor se carga muy lentamente provocando un comportamiento como de circuito abierto (CA) impidiendo el paso de la corriente hacia el interior de la célula, en consecuencia la impedancia medida se debe únicamente al componente resistivo del espacio extracelular. Por otro lado, cuando se inyecta corriente de alta frecuencia, la velocidad de carga del capacitor aumenta creando un efecto de corto circuito (CC) permitiendo que la corriente atraviese la membrana celular hasta el espacio intracelular, por lo tanto la impedancia medida es producto del componente resistivo extracelular e intracelular, este comportamiento se ilustra en las figuras 9 y 10.

a)

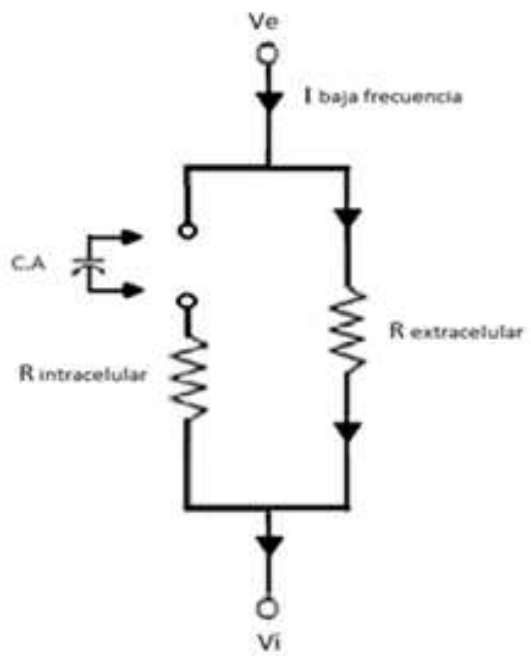

b)

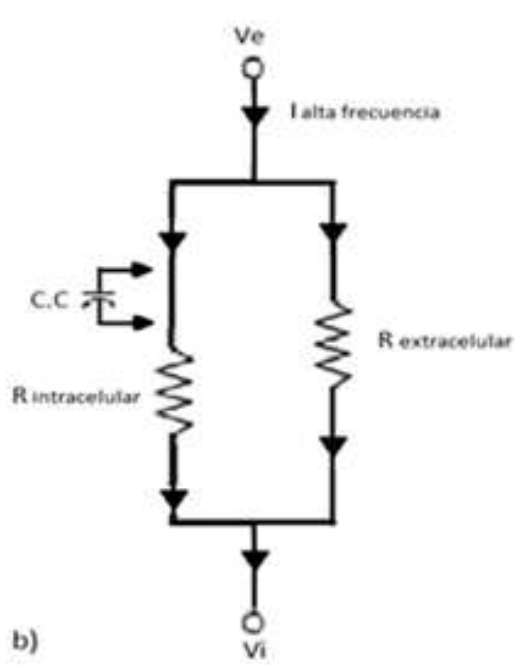

Figura 9. Comportamiento eléctrico de la célula al paso de corriente alterna, a) paso de la corriente de baja frecuencia permitiendo que el capacitor se comporte como un circuito abierto (C.A) y b) paso de la corriente de alta frecuencia creando un efecto de corto circuito (C.C) en el capacitor. 


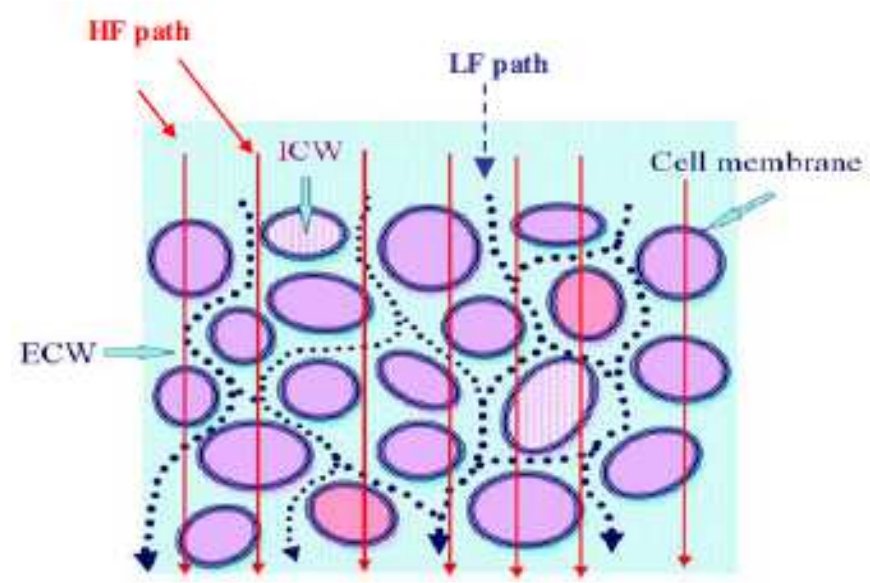

Figura 10. Trayectorias de la corriente en el tejido a bajas y altas frecuencias, las líneas rojas corresponden a la trayectoria de la corriente a alta frecuencia mientras que la línea punteada marca el camino de la corriente de baja frecuencia.[14]

\subsection{Bioimpedancia y sus clasificaciones}

El BIA tiene cuatro variantes: BIA mono frecuencia, BIA multifrecuencia, espectrometría de bioimpedancia (EBI) y BIA vectorial. Para el BIA de mono frecuencia se utiliza a una frecuencia fija de $50 \mathrm{kHz}$, la cual permite únicamente la estimación de agua corporal total (TBW o ACT); mientras que el BIA de multifrecuencia realiza un barrido de frecuencias desde $1 \mathrm{kHz}$ hasta $10 \mathrm{MHz}$, permitiendo la estimación del ACT y el volumen extracelular (VEC) a partir de los cuales se obtiene el volumen intracelular (VIC) [10,15].

El BIA vectorial (BIAV) es una variación del BIA monofrecuencia; esta técnica se basa en la representación gráfica del vector de impedancia para obtener una valoración semicuantitativa del estado hídrico del paciente. Se gráfica el vector de impedancia promedio contra la variabilidad de la población de referencia la cual esta descrita por varias elipses concéntricas de tolerancia. Las elipses son específicas para cada género y raza [2].

La espectroscopia de bioimpedancia (EBI). Al igual que el BIA de multifrecuencia realiza un barrido de frecuencias ( $1 \mathrm{kHz}$ a $1 \mathrm{MHz}$ ) con la diferencia de que efectúa el ajuste complejo de la impedancia a un modelo matemático mejor conocido como modelo Cole-Cole; tomando en cuenta las componentes reales e imaginarias de la impedancia [16]. Como se muestra en la figura 11 a frecuencia cero o DC la impedancia se debe únicamente a resistencia, conforme la frecuencia aumenta la reactancia aumentan hasta llegar a su punto máximo, a partir de este punto aunque la frecuencia siga aumentando la reactancia empiezan a disminuir hasta que la reactancia se vuelve cero y la impedancia medida corresponde totalmente a los componentes resistivos, por lo que la impedancia tiene únicamente dos puntos en los cuales interseca el eje real, uno a DC y el otro a frecuencia infinita. 


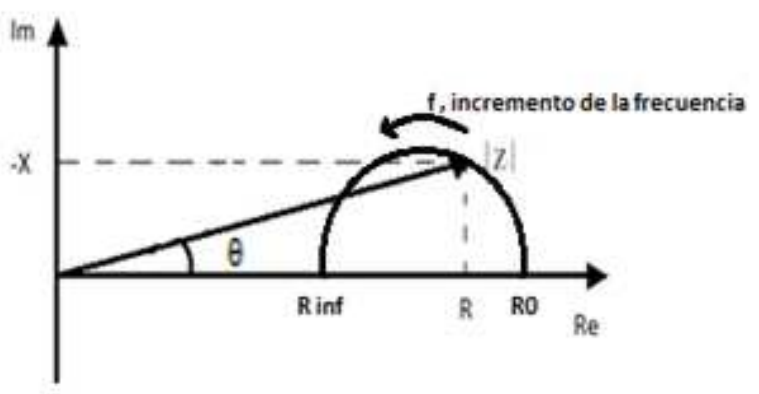

Figura 11. Modelo de impedancia de Cole-Cole.

\subsection{Bioimpedancia en Hemodiálisis}

El uso de la bioimpedancia en HD se ha ramificado en cuatro líneas de interés: identificación del peso seco (ACT), determinación de los cambio en los volúmenes entre compartimentos durante la sesión, consideración del volumen de distribución de la urea para el cálculo del Kt/V y valoración nutricional (masa magra, masa grasa) [15]. En este sentido dependiendo de la aplicación se ha evaluado el desempeño clínico del BIA mono y multi-frecuencia, BIAV y EBI en diferentes condiciones que van desde realizar mediciones de impedancia antes y/o después de la sesión de hemodiálisis y variando la colocación de los electrodos para conseguir un análisis de cuerpo completo o segmentado [16-18]. Para el caso de los estudios de cuerpo completo se ha observado que no es posible obtener mediciones precisas, pues existen demasiados errores debidos a que la distribución de líquidos en el cuerpo es diferente para cada persona (composición corporal); sin embargo se ha llegado a la conclusión de que aproximadamente el $90 \%$ de la resistencia obtenida por el BIA de cuerpo completo proviene de los brazos y las piernas [3].

Existen múltiples estudios que realizan mediciones segmentadas, ya sea de medio cuerpo (lado izquierdo o derecho), brazo, pierna e incluso el tronco. Las mediciones de medio cuerpo muestran en general el mismo efecto que las mediciones de cuerpo completo, es decir, dependen de la cantidad de líquido que se encuentra en las extremidades, por esta razón cada vez son más utilizados los estudios segmentados ya sea de brazo o pierna. En el 2006 Fasan Zhun realizó un estudio donde obtuvo el estado hídrico del paciente realizando mediciones de BIA en la pantorrilla; bajo la premisa de que es en las piernas en específico la parte baja de las piernas donde se encuentra la mayor acumulación de líquido debido a efectos gravitatorios. En este estudio se considera la pantorrilla como un cilindro cuya circunferencia disminuye conforme el exceso de líquido es retirado, por lo que es necesario medir la circunferencia de la pantorrilla antes de efectuar la medición para ajustar el modelo al nuevo volumen del cilindro lo cual complica su implementación para medir de forma continua[3].

En el 2011 Guillermo Medrano realizó otro estudio donde sugiere medir de forma segmentada en las piernas usando un circuito que hace el recorrido eléctrico de rodilla a rodilla, este método a pesar de nunca antes había sido utilizado reportó una buena reproducibilidad con variaciones de 
1.6 y $2.6 \%$ para la resistencia extra e intracelular respectivamente [11]. Cabe señalar que este método aún no ha sido comparado contra el estándar de oro (métodos por dilución).

Los estudios mencionados anteriormente muestran que es posible estimar el estado hídrico de los pacientes mediante bioimpedancia; sin embargo, son pocos los estudios que proponen la medición de impedancia de manera continua durante toda la sesión, debido a la gran cantidad de artefactos a los que se someten la resistencia y la impedancia. Por esta razón la mayor parte de los estudios son realizados colocando al paciente en posición supina procurando que las extremidades no estén en contacto con ninguna otra parte del cuerpo y procurando que tanto la ropa como la superficie donde se encuentra el paciente no sean conductivas [17].

Existen pocos estudios que realizan la espectroscopia de bioimpedancia durante el tratamiento. No obstante, en el 2011 Gabriela Béltran muestra que es posible correlacionar el cambio en el volumen del LIC y el LEC mediante espectroscopía de reactancia durante la sesión de hemodiálisis, sin embargo, se encontró una alta variabilidad de las mediciones debidas a diferentes fuentes de ruido así como artefactos de movimiento [19]. A pesar de que esta técnica es capaz de estimar el estado hídrico del paciente, aún existe una gran variabilidad en las mediciones inter e intra paciente, la cual ha impedido que se establezca un rango de valores que limiten la cantidad de líquido a extraer por sesión.

La literatura también sugiere que existe un rebote eléctrico que puede provocar una sobre estimación en la medición de la impedancia ocasionado por un estado de desequilibrio de la urea, una de las teorías para el rebote eléctrico se basa en que es más sencillo llevar a cabo la remoción de urea del LEC, el cual es extraído durante el tratamiento provocando una disminución en la concentración de urea extracelular; en consecuencia, al finalizar el tratamiento se forma un gradiente de concentración que a su vez provoca el flujo de urea del LIC al LEC hasta que la concentración de urea alcance un nuevo punto de equilibrio. El rebote eléctrico puede ser evitado si la medición de impedancia se realiza 30 minutos después del tratamiento [18]. Pese a esto se desconoce realmente la causa del rebote eléctrico y sí realmente afecta la estimación de la impedancia [20,21].

Como se menciono anteriormente, las mediciones intradiálisis no se realizan comúnmente debido a la gran cantidad de artefactos que afectan la medición. Sin embargo al entender la fuente de los artefactos es posible prevenirlos o bien disminuir el impacto de los mismos. Es decir, se ha demostrado que la medición de BIA de cuerpo completo está más expuesta a producir artefactos de línea debido a que la disposición de los electrodos y cables de registro actúan como antenas. Por lo que sí se quiere eliminar este artefacto bastará con disminuir la distancia entre electrodos, es decir, realizar la medición de manera segmentada en vez de la de cuerpo completo otorgará una mejor relación señal a ruido debido a que se reduce el efecto antena por parte del paciente y de los cables de registro. Aunado a esto, está demostrado que la mayor contribución a la impedancia corporal viene de las extremidades en particular de los muslos y los antebrazos. 


\section{Ericka Stanford Alcántara}

De ahí, resultaría evidente que para realizar mediciones continúas durante la diálisis se diseñaran dispositivos capaces de medir en las piernas ya que son estas las que más contribuyen a la impedancia total, sin embargo, durante los tratamientos de hemodiálisis es común que los pacientes que presentan cuadros de hipotensión sintomática se les eleven las piernas como maniobra para aumentar el retorno venoso y contrarrestar la hipotensión; o bien en algunas clínicas de hemodiálisis los pacientes realizan ejercicio continuo en ergómetros durante la sesión. Estos dos factores suponen una fuente importante de ruido debido al cambio de posición y al movimiento producido durante el ejercicio, por lo que la medición en las piernas se vería afectada bajo estas condiciones. Por el contrario, el antebrazo se encuentra menos expuesto a estos artefactos y es la segunda región que más contribuye a la impedancia corporal total [11].

Por lo que el registro de BIA en el antebrazo debería minimizar los problemas de registro debidos a los artefactos de movimiento, cambios de posición, sobre estimación hídrica debida a efectos gravitatorios, antenas y al ruido de línea. No obstante, también se debe de mejorar la disposición de los electrodos de tal forma que se minimicen variaciones en cuanto a la colocación de los electrodos. Ya que la forma en la cual se colocan los electrodos también contribuye a la variabilidad intra e interpaciente. Por lo que se debe estandarizar estas mediciones para poder garantizar su reproducibilidad. Existen dos formas para eliminar la variabilidad debida a los electrodos; la primera corresponde a colocar los electrodos en el mismo lugar cada vez que se realice la medición y en segundo garantizar que la distancia entre electrodos sea la misma. De esta manera se homogenizaria la colocación de los electrodos, sin embargo, es difícil garantizar que siempre se coloquen los electrodos en el mismo lugar, pero, si es posible asegurar que la distancia entre electrodos sea la misma. En este sentido estamos hablando de asegurar una geometría fija entre electrodos.

La geometría fija se consigue al montar los electrodos en una base rígida que no permita deformaciones que alteren la distancia entre electrodos y más importante, se garantiza que la región transversal por la que fluye la corriente sea la misma intra e interpaciente. Por lo que un arreglo de electrodos de geometría constante diseñado para el antebrazo promete mejorar la condición de registro del BIA de tal forma que logren mediciones reproducibles durante la sesión de hemodiálisis, permitiendo monitorizar el equilibrio hídrico del paciente durante toda la sesión y no solo antes y después como se realizan actualmente. 


\subsection{Espectrómetro de impedancia}

Se utilizó un espectrómetro de impedancia que mide la impedancia compleja en un rango de 23 frecuencias que abarcan desde $215 \mathrm{~Hz}$ a $1 \mathrm{kHz}$ a través del método de demodulación paralela y conversión digital propuesto por Othman y Sacristán [22]. El equipo cumple con estándares de regulación internacionales como la BS EN 60601-1:1990 y la ANSI/AAMI ES1:1993. Se diseño un arreglo de electrodos para el antebrazo que permite la adquisición de la impedancia eléctrica.

El arreglo de electrodos consta de cuatro electrodos de superficie, donde los dos electrodos exteriores inyectan la corriente de excitación al tejido, dicha corriente genera un voltaje el cual es medido por los dos electrodos internos. Para garantizar que la toda la corriente fluya por el tejido la impedancia de entrada del sistema debe ser mayor en orden de magnitud que la impedancia del tejido medido y que la impedancia de la interfase electrodo-pie. De esta manera la impedancia total representa la impedancia del tejido únicamente [9].

El espectrómetro utiliza una interfase con una computadora para el almacenamiento, procesamiento y despliegue de información. 
Estimación del equilibrio hídrico tisular en hemodiálisis mediante espectroscopía de reactancia Ericka Stanford Alcántara 


\section{Materiales y Métodos}

Como se explicó en la sección anterior, actualmente no existe un dispositivo de EBI capaz de realizar mediciones intradiálisis sin verse afectado por interferencias debidas a factores externos y factores propios del tratamiento. En esta sección se presentan los materiales y métodos utilizados para probar un arreglo de electrodos que minimiza las interferencias permitiendo las mediciones de EBI durante hemodiafiltración.

\subsection{Ubicación de electrodos}

Se seleccionó el antebrazo como la zona anatómica de colocación del arreglo de electrodos, partiendo de la premisa de que la mayor cantidad de fluidos corporales se encuentran en las extremidades y no en el torso.

Sabemos que el musculo es el tejido que posee más fluidos en comparación con el hueso y el tejido adiposo; en este sentido si se busca encontrar diferencias en el equilibrio hídrico sería intuitivo colocar los electrodos en la zona de mayor musculatura, en las extremidades inferiores. Sin embargo, se descartó esta posibilidad ya que las extremidades inferiores están más expuestas que las extremidades superiores a los artefactos de movimiento (en caso de realizar ejercicio transdiálisis) y cambios de posición que puedan modificar la distribución de líquidos; recordando que la maniobra más común en HDF para disminuir los síntomas de la hipotensión consiste en elevar las piernas del paciente para aumentar el retorno venoso, los cuales podrían afectar la medición.

Quedando como alternativa las extremidades superiores, finalmente se optó por el antebrazo. Considerando que esta zona tiene una menor acumulación de tejido graso comparado con la parte superior del brazo y la circunferencia entre pacientes no varía tanto, lo que permite construir un electrodo con medidas estándar.

\subsection{Electrodos de Ag-AgCl}

Al inicio se quería que el arreglo de electrodos fuera lo más pequeño posible para evitar molestias al paciente, para ello se construyeron electrodos de hilo de $\mathrm{Ag}-\mathrm{AgCl}$ montados en un semicírculo de acrílico, sin embargo, no se pudo lograr una buena interfase electrodo-electrolito-piel. Debido a que no se pudo garantizar una superficie de contacto uniforme. Por lo que se escogió el uso de electrodos comerciales desechables para ECG de gel sólido marca 3M. A estos electrodos únicamente se les removió el soporte de espuma adhesiva, dejando el gel conductor y el broche para que puedan ser colocados en el soporte mencionado a continuación. 


\section{Ericka Stanford Alcántara}

\subsection{Geometría fija entre electrodos.}

Parte importante de este proyecto consistió en buscar la repetitividad de las mediciones. Para esto fue necesario eliminar una fuente importante de error, es decir, la colocación de los electrodos. En la colocación de los electrodos intervienen dos factores importantes: 1) el sitio de colocación y 2) la distancia entre electrodos. Si bien es difícil garantizar que los electrodos se coloquen siempre en el mismo punto del antebrazo, si podemos garantizar la distancia que existe entre ellos. Para esto, se fijaron broches para electrodos estándar en medio brazalete de acrílico de $13 \times 7 \mathrm{cms}$. Con una separación de $11 \mathrm{cms}$ entre los electrodos de inyección y $4.5 \mathrm{cms}$ entre electrodos de medición ver figura 12

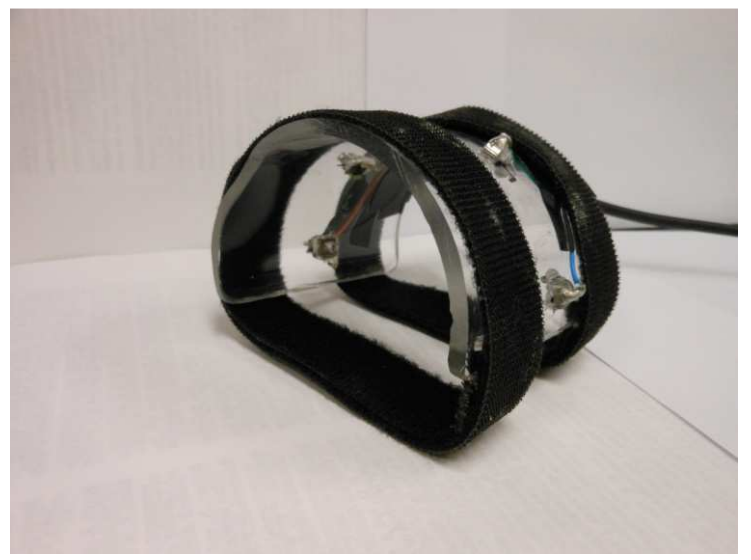

Figura 12. Imagen del arreglo de electrodos.

\subsection{Población en Hemodiafiltración.}

Los estudios se realizaron en la unidad de hemodiálisis del Instituto Nacional de Cardiología "Dr. Ignacio Chavéz" (INC). Se estudiaron 4 pacientes (3 mujeres y 1 hombre) de entre 18-38 años con IRC estadio 5 que reciben tratamiento renal sustitutivo mediante hemodiafiltración (HDF). Los pacientes no reciben medicamentos antihipertensivos, antihistamínicos, antidepresivos o antiespasmódicos y no se encuentran sometidos bajo ningún régimen nutricional.

\subsubsection{Criterios de inclusión}

- Pacientes con IRC estadio 5.

- Edad mayor a 17 años y menor a 60 años.

- Recibir tratamiento de HDF 3 veces a la semana con una duración no menor a 3 hrs.

- Estancia mayor a 1 mes en el programa de HDF.

- Acceso vascular mediante catéter. 


\subsubsection{Criterios de exclusión}

- Pacientes con enfermedades asociadas: diabetes mellitus, cardiopatías, hiperparatiroidismo o neuropatías.

- Pacientes inestables, con hipotensión arterial en más del $25 \%$ de sus sesiones anteriores.

- Enfermedades autoinmunes.

\subsubsection{Procedimiento}

La medición de espectroscopia de impedancia se realizó mediante la colocación del arreglo de electrodos en la parte inferior del antebrazo del lado opuesto al catéter y al brazalete de insuflación como se muestra en la figura 13, para evitar que las mediciones se vieran afectadas por los cortes del flujo sanguíneo producto del brazalete de insuflación o a una posible recirculación y/o falta de irrigación debida al catéter. La zona del antebrazo donde se colocó el arreglo de electrodos se limpió previamente con alcohol.

El estudio se llevó a cabo en 4 sujetos en tres sesiones de HDF diferentes, cada espectro o medición de $E B I$ corresponde a un barrido de frecuencias de $215 \mathrm{~Hz}-1 \mathrm{MHz}$ inyectando una corriente de $1 \mathrm{~mA}$. La adquisición de espectros se realizó cada 15 minutos durante toda la sesión suponiendo que en un intervalo de tiempo menor no existen cambios hídricos significativos. Para cada intervalo de tiempo se adquirieron tres espectros inmediatamente consecutivos con la finalidad de promediarlos y disminuir variaciones.

La primer medición o el primer espectro se obtiene antes de que inicie la sesión, con el paciente sentado en el lugar donde recibirá el tratamiento; la segunda medición se realiza transcurridos 15 minutos de tratamiento y así sucesivamente para cada una de las mediciones con excepción de la última (ver figura 14). Generalmente la duración de la sesión de HDF resulta ser un múltiplo de 15 minutos por lo que la última medición coincide exactamente con el fin de la sesión y el inicio del protocolo de desconexión del paciente, lo cual provoca mucho movimiento del paciente dificultando la medición. Por tanto, se optó por realizar la última medición en el instante en el que el paciente es desconectado.

La información adquirida con el espectrómetro de impedancia fue almacenada en una PC que tenía previamente cargado el software especializado. 


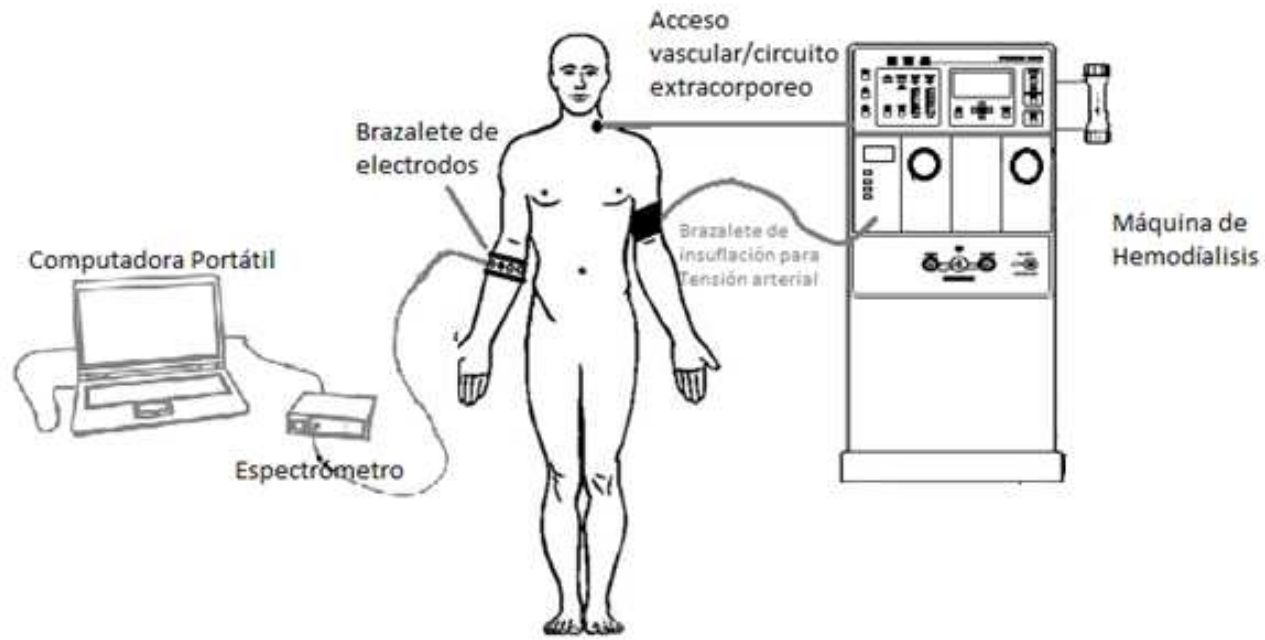

Figura 13. Esquema de colocación del arreglo de electrodos de forma contra lateral al acceso vascular y al brazalete de insuflación [8].

\subsubsection{Instrumentación}

- Espectrómetro de impedancia marca Innovamédica.

- Arreglo de electrodos.

- Electrodos desechables para ECG maraca 3M.

- Computadora portátil.

- Equipo de Hemodiálisis marca Fresenius Medical Care, modelo 4008H.

- Filtros de polisulfona de alto flujo F80A.

\subsubsection{Análisis de Datos}

Para obtener un solo espectro por medición se promediaron los espectros de todos los pacientes cada 30 y 60 minutos, la agrupación de los intervalos de tiempo se hizo a partir de la última medición hacia atrás hasta llegar al principio de la sesión con el fin de evaluar los cambios en reactancia a partir del supuesto equilibrio hídrico alcanzado al final de la sesión.

Se calcularon los parámetros de Cole-Cole para los espectros obtenidos y se realizó el análisis de varianza de medidas repetidas (ANOVA) para la reactancia central a altas frecuencias por intervalos de tiempo de 15 minutos. Se consideraron diferencias estadísticamente significativas cuando $\mathrm{p}<0.01$.

\subsection{Población de Voluntarios Sanos}

Se estudiaron 4 sujetos sanos (3 hombres y 1 mujer) de entre 24-33 años. Los voluntarios no reciben medicamentos y no se encuentran sometidos bajo ningún régimen nutricional. Los estudios se realizaron en el laboratorio de instrumentación del Centro Nacional de Investigación en Instrumentación e Imagenología Médica ( $\mathrm{Cl}^{3} \mathrm{M}$ ) en la UAM-Iztapalapa. 


\subsubsection{Criterios de inclusión}

- Edad mayor a 17 años y menor a 60 años.

\subsubsection{Criterios de exclusión}

- Tener un $\mathrm{IMC} \geq 30$.

- Enfermedades cardiovasculares y/o metabólicas.

- 8 hrs $<$ Ayuno $<12$ hrs

\subsubsection{Criterios de rechazo}

- Tiempo para realizar la ingesta de agua mayor a 10 minutos.

\subsubsection{Procedimiento}

Se les instruyo a los voluntarios que mantuvieran un ayuno mínimo de 8 hrs y máximo de 12 hrs tanto de bebidas como de alimentos. Se pesó a los voluntarios en una báscula digital al principio y al final del estudio. Se mantuvieron sentados durante la totalidad del estudio.

Se limpió con alcohol la zona donde se colocó el arreglo de electrodos. Para cada uno de los voluntarios se obtuvieron 3 espectros de reactancia para cada una de las siguientes condiciones (ver figura 14):

- Ayuno: Las mediciones se realizaron una vez colocado el arreglo de electrodos.

- Ingesta de Agua: Mediciones obtenidas después de ingerir 1 litro de agua embotellada.

- 1 hora después de la ingesta: Mediciones obtenidas transcurridos 60 minutos después de la ingesta de agua.

\subsubsection{Instrumentación}

- Espectrómetro de impedancia marca Innovamédica.

- Arreglo de electrodos.

- Electrodos desechables para ECG maraca 3M.

- Computadora portátil.

- Báscula digital con capacidad máx. $180 \mathrm{Kg}$.

\subsubsection{Análisis de Datos}

Se calcularon los parámetros de Cole-Cole para los espectros obtenidos y se realizó un análisis de varianza de medidas repetidas (ANOVA) para la reactancia central a altas frecuencias con un valor de significancia de $p<0.05$. 
Estimación del equilibrio hídrico tisular en hemodiálisis mediante espectroscopía de reactancia

\section{Poblaciones}

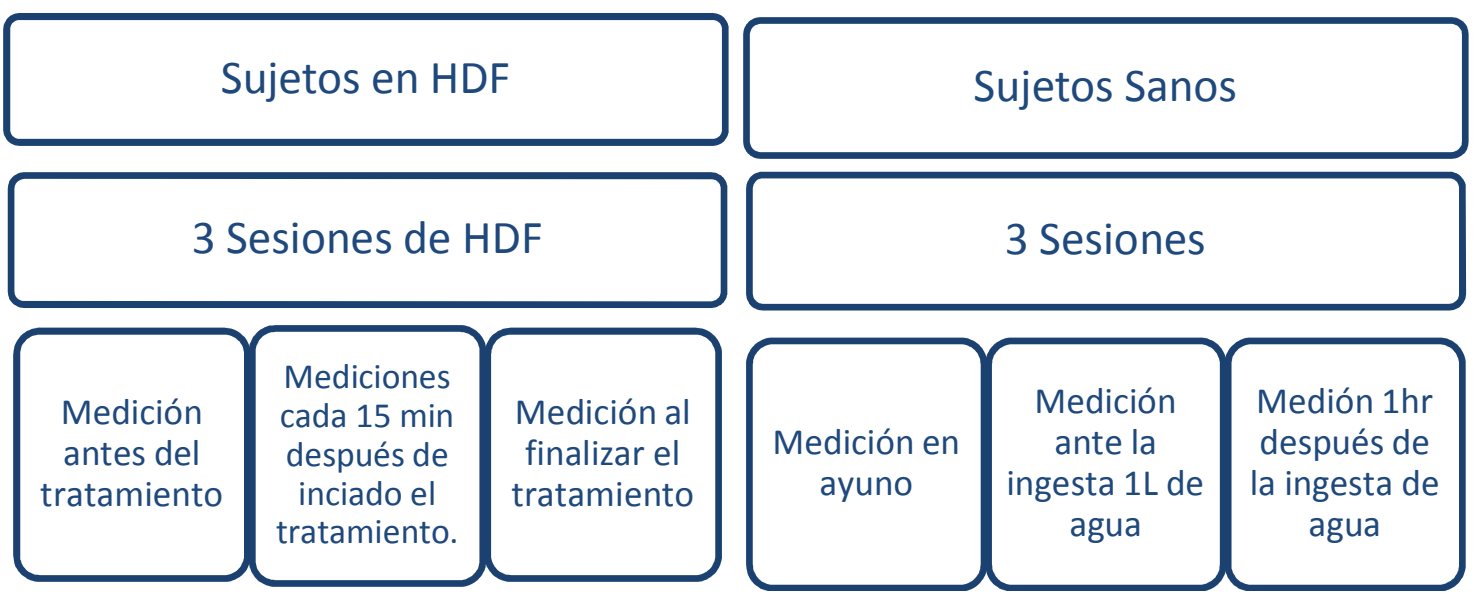

Figura 14. Diagrama de la metodología empleada para cada población. 


\section{Resultados}

\subsection{Descripción de la población en HDF}

En la tabla 2 se muestra la talla y el peso promedio de cada sujeto pre y pos diálisis; Así como la diferencia de pérdida de peso por paciente. Se observa que existe una pérdida de peso mínima de $2.5 \mathrm{~kg}$ y máxima de $4.5 \mathrm{~kg}$. Recordando que dicha pérdida de peso es ocasionada por la extracción de líquido y toxinas del cuerpo durante la hemodiálisis en un tiempo no mayor a 4 hrs. Lo que hace a este tratamiento bastante agresivo.

\begin{tabular}{ccccc}
\hline \multicolumn{5}{c}{ Tabla 2. Peso y talla de la población en HDF } \\
\hline Paciente & Talla & Peso Pre-HDF & Peso Pos-HDF & Pérdida de peso \\
1 & $1.71 \mathrm{~m}$ & $58.70 \pm 1.25 \mathrm{~kg}$ & $54.17 \pm 1.53 \mathrm{~kg}$ & $4.53 \pm 0.45 \mathrm{~kg}$ \\
2 & $1.52 \mathrm{~m}$ & $52.53 \pm 0.99 \mathrm{~kg}$ & $49.93 \pm 0.83 \mathrm{~kg}$ & $3.00 \pm 0.2 \mathrm{~kg}$ \\
3 & $1.63 \mathrm{~m}$ & $74.73 \pm 0.12 \mathrm{~kg}$ & $71.73 \pm 0.46 \mathrm{~kg}$ & $3.00 \pm 0.53 \mathrm{~kg}$ \\
4 & $1.57 \mathrm{~m}$ & $61.27 \pm 0.06 \mathrm{~kg}$ & $58.77 \pm 0.32 \mathrm{~kg}$ & $2.50 \pm 0.34 \mathrm{~kg}$ \\
\hline
\end{tabular}

En la siguiente tabla se muestra el índice de masa corporal (IMC) de los pacientes. Ya que los pacientes presentan diferentes pesos en cada sesión, así como al inicio y al final de cada tratamiento; Lo que se muestra en la tabla 3 es el IMC obtenido mediante el peso promedio de los pacientes pre y pos HDF.

Cabe destacar el uno de los criterios de exclusión para este protocolo consistía en no presentar un $I M C>30$, a partir de IMC mayores a este valor se considera que la persona presenta algún grado de obesidad.

\begin{tabular}{|c|c|c|}
\hline \multicolumn{3}{|c|}{$\begin{array}{c}\text { Tabla 3.Índice de masa corporal (IMC) promedio de la } \\
\text { población en HDF }\end{array}$} \\
\hline Paciente & Pre-HDF & Pos-HDF \\
\hline 1 & $20.07 \pm 0.43 \mathrm{~kg} / \mathrm{cm}^{2}$ & $18.52 \pm 0.52 \mathrm{~kg} / \mathrm{cm}^{2}$ \\
\hline 2 & $22.74 \pm 0.43 \mathrm{~kg} / \mathrm{cm}^{2}$ & $21.61 \pm 0.36 \mathrm{~kg} / \mathrm{cm}^{2}$ \\
\hline 3 & $28.13 \pm 0.04 \mathrm{~kg} / \mathrm{cm}^{2}$ & $27.00 \pm 0.17 \mathrm{~kg} / \mathrm{cm}^{2}$ \\
\hline 4 & $24.86 \pm 0.02 \mathrm{~kg} / \mathrm{cm}^{2}$ & $23.84 \pm 0.13 \mathrm{~kg} / \mathrm{cm}^{2}$ \\
\hline
\end{tabular}




\subsection{Espectros típicos.}

Se graficaron los espectros de resistencia y reactancia promedio de todos los tratamientos. De esta manera es posible detectar si el dispositivo es capaz de realizar mediciones de EBI fiables con el arreglo de electrodos diseñado para el antebrazo cuando el paciente se encuentra en tratamiento de HDF. En la gráfica 1a se muestra el espectro de resistencia, el cual disminuye conforme aumenta la frecuencia. Mientras que en la gráfica $1 \mathrm{~b}$ se observa que el espectro de reactancia permanece constante a bajas frecuencias y al aumentar la frecuencia se crea un lóbulo que corresponde a la región de dispersión beta. Para estas gráficas no se incluye el error estándar ya que el propósito de las mismas es ilustrar el comportamiento de resistencia y reactancia

a)

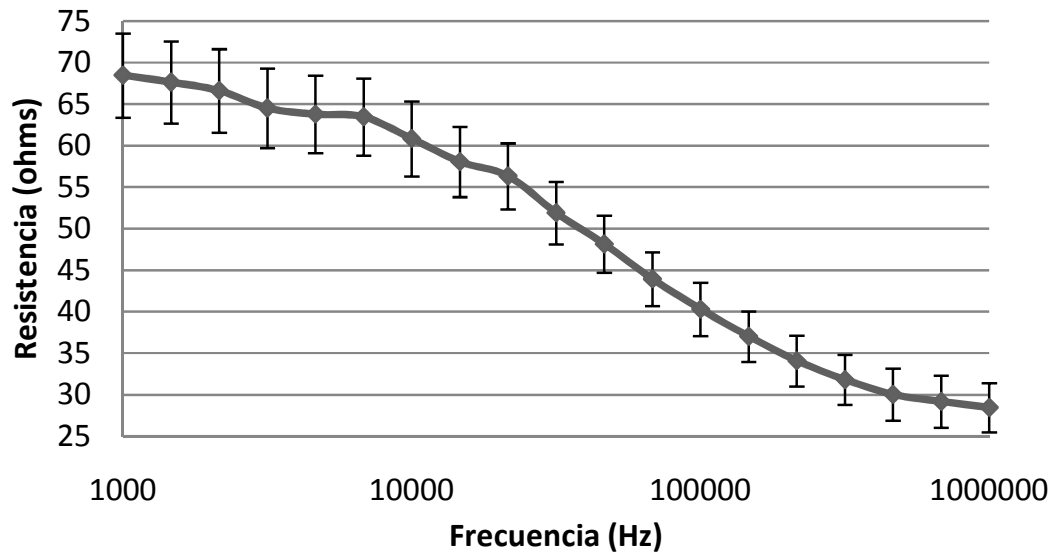

b)

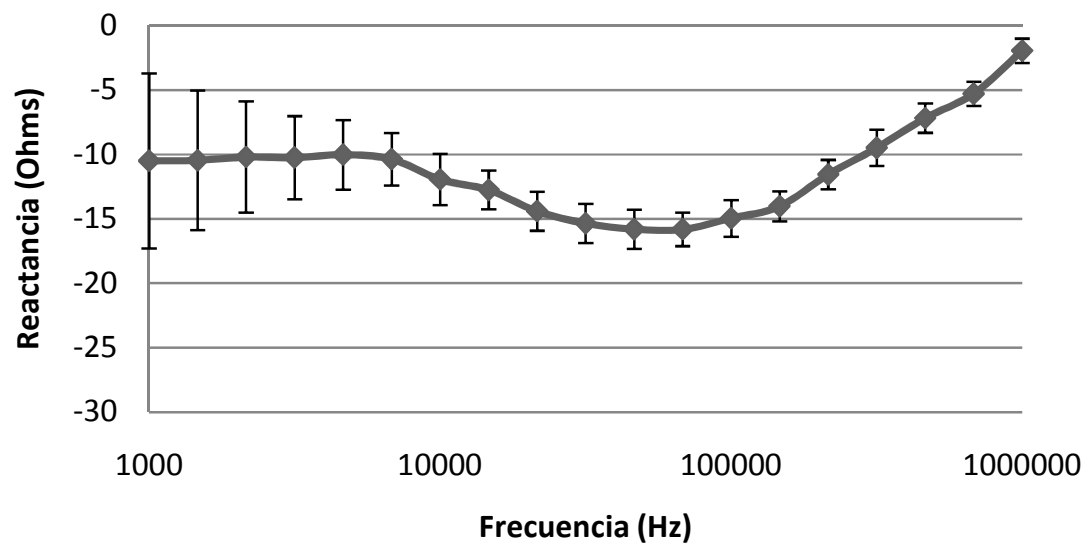

Gráfica 1.Espectros de reactancia promedio de todos los pacientes $(n=12) \pm$ error estándar:

a) espectro de resistencia y b) espectro de reactancia. 


\section{Ericka Stanford Alcántara}

Una forma de visualizar la información de impedancia es mediante la gráfica de Nyquist en donde se grafica la resistencia vs la reactancia negativa. La gráfica resultante genera un semicírculo, a partir del cual el modelo de Cole-Cole caracteriza la bioimpedancia.

La gráfica 2 muestra el diagrama de Nyquist obtenido a partir de los espectros anteriores de resistencia y reactancia. La línea punteada corresponde al semicírculo ajustado a partir del modelo de Cole-Cole, el cual realiza el ajuste de los parámetros de bioimpedancia al semicírculo que mejor se aproxime a los valores experimentales. Y los puntos son datos experimentales. De esta gráfica se obtiene que la reactancia máxima es de 15.50 -j $\Omega$ mientras que la resistencia es de 46.91 $\Omega$ con un error de $0.014 \Omega$; según los parámetros obtenidos del software del espectrómetro.

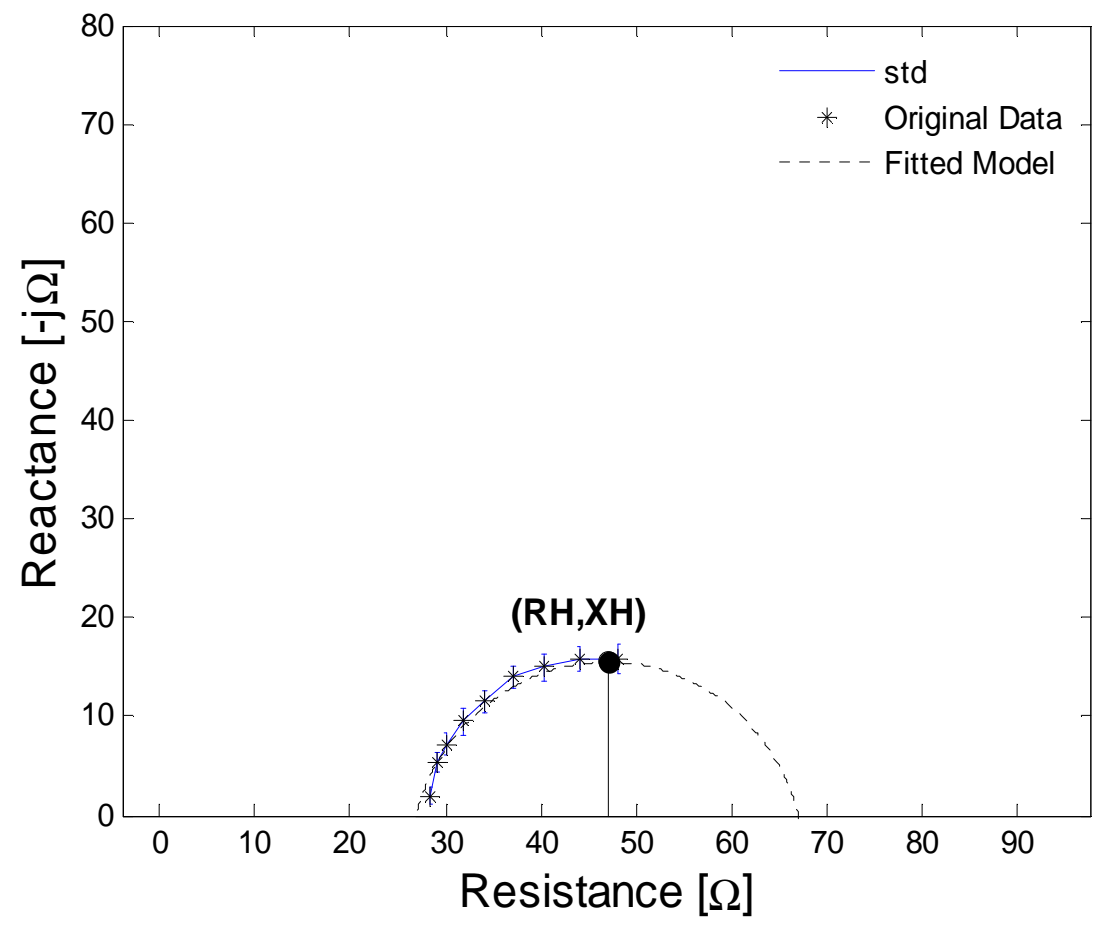

Grafica2. Semicírculo ajustado de Cole-Cole y datos medidos con desviación estándar ( $\mathrm{n}=12$ ) . Donde (Rh,Xh) corresponde al punto donde se alcanza la reactancia máxima para altas frecuencias.

La gráfica anterior únicamente muestra el diagrama de Nyquist para altas frecuencias, para propósitos de este proyecto únicamente se trabajó con altas frecuencias ( $f>50 \mathrm{khz}$ ) debido a que el lóbulo de reactancia se forma a partir de los $80 \mathrm{Khz}$ aproximadamente (ver gráfica $1 \mathrm{~b}$ ) además de que se pretendió eliminar posibles fuentes de interferencia ya que estas afectan principalmente a los componentes de baja frecuencia. 


\section{Ericka Stanford Alcántara}

\subsection{Comportamiento de reactancia en HDF.}

En la sección anterior se observó la viabilidad de las mediciones de EBI en el antebrazo durante HDF. Parte fundamental de este trabajo consiste en buscar cambios de reactancia conforme se remueve el exceso de agua durante el tratamiento; por lo que en esta sección se realizó un análisis más detallado de la reactancia en el tiempo.

Para el análisis de los espectros de reactancia se consideró la duración de las sesiones de HDF ya que son diferentes tanto intra como inter paciente provocando que los espectros obtenidos del promedio de cada paciente tuviesen duraciones distintas, por lo que se utilizaron únicamente los lapsos de tiempo que contaron con información completa de todos los pacientes, de esta forma se estandarizó la duración de la sesión hasta los 195 minutos, los datos correspondientes a tiempos posteriores no se tomaron en cuenta para la obtención de los espectros de reactancia. Por lo que cada sesión consta de 14 espectros adquiridos cada 15 minutos desde el tiempo 0 hasta los 195 $\min$.

Como consideración adicional se agruparon los espectros de reactancia desde el final hacia el principio del tratamiento tomando como tiempo de inicio o tiempo 0 la última medición realizada al paciente permitiendo que el tiempo transcurra de manera negativa. Se aplicó esta consideración partiendo del hecho de que al final del tratamiento todos los pacientes debieron de alcanzar su equilibrio hídrico mientras que al inicio cada paciente debería presentar diferentes grados de hidratación (debidos a diferencias en ingesta de líquidos y comidas, así como actividad física); por lo que resulta evidente alinear los datos a partir del punto de supuesto equilibrio hídrico y no desde el inicio de la sesión.

Se promediaron los espectros de resistencia y reactancia de todos los pacientes en intervalos de tiempo de una hora para visualizar el cambio de reactancia en el tiempo. En la gráfica 3 se presentan los diagramas de Nyquist para tres intervalos de tiempo: 0 min, -60 min y -120 min. 
Ericka Stanford Alcántara

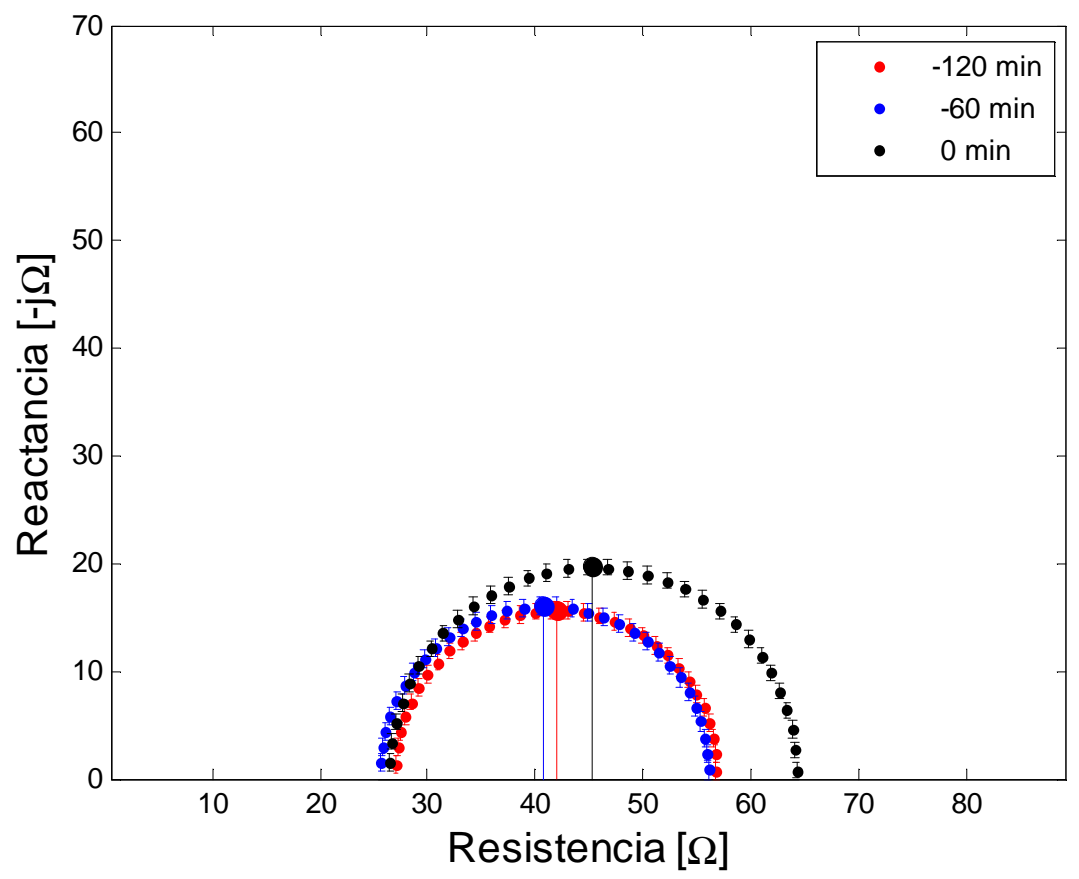

Gráfica 3. Espectro de reactancia promedio \pm desviación estándar de los pacientes en HDF ( $n=12)$, la curva en color negro corresponde al tiempo cero, la azul a -60 minutos y en rojo -120 minutos.

\subsection{Reactancia central durante HDF.}

Recordando que uno de los objetivos es observar cambios en la reactancia durante la sesión de HDF, se obtuvieron a partir de los parámetros del modelo de Cole-Cole las reactancias centrales para altas frecuencias $(\mathrm{Xh})$ ya que los lóbulos obtenidos de mayor amplitud se encuentran en frecuencias mayores a $50 \mathrm{KHz}$. Se graficó la Xh promedio de todos los pacientes para cada intervalo de tiempo (15 minutos). Se tomaron las mismas consideraciones para realizar las gráficas que las utilizadas en los espectros de reactancia. En la gráfica 4 se presenta el cambio en reactancia central promedio a lo largo del tiempo con el error estándar (error estándar $<0.5$ ohms). 


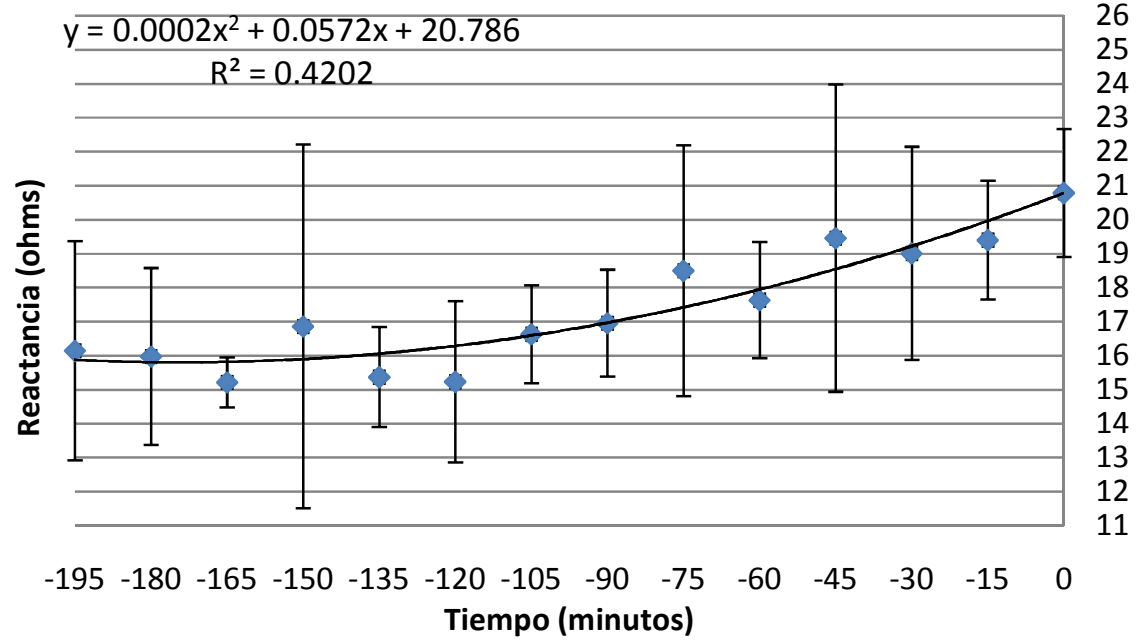

Gráfica 4. Cambio en reactancia central a altas frecuencias en el tiempo, cada punto está representado como media \pm desviación estándar.

Para evaluar si existen cambios de reactancia en el transcurso del tratamiento se realizó un ANOVA de medidas repetidas, considerando resultados estadísticamente significativos con una $p<0.01$. Se encontró una diferencia estadísticamente significativa $(p=0.008511)$ de la reactancia en el tiempo.

\subsection{Reactancia en Sujetos Sanos.}

Para poder determinar si la reactancia central de los pacientes corresponde al equilibrio hídrico es importante conocer el valor de reactancia que corresponde el equilibrio hídrico en sujetos sanos, en los cuales no existe ninguna patología que pudiera alterar dicho equilibrio. Para esto se realizaron mediciones de espectroscopia de impedancia en sujetos sanos durante 3 situaciones (ayuno, ingesta de agua, después de la ingesta), a través de las cuales se evaluó el cambio en reactancia ante el reajuste del equilibrio hídrico.

Se observaron cambios en el espectro de reactancia ante la ingesta de 1 litro de agua natural embotellada. Para esto se promediaron los espectros de reactancia de los cuatro voluntarios para cada condición de registro como se muestra en la gráfica 5. 


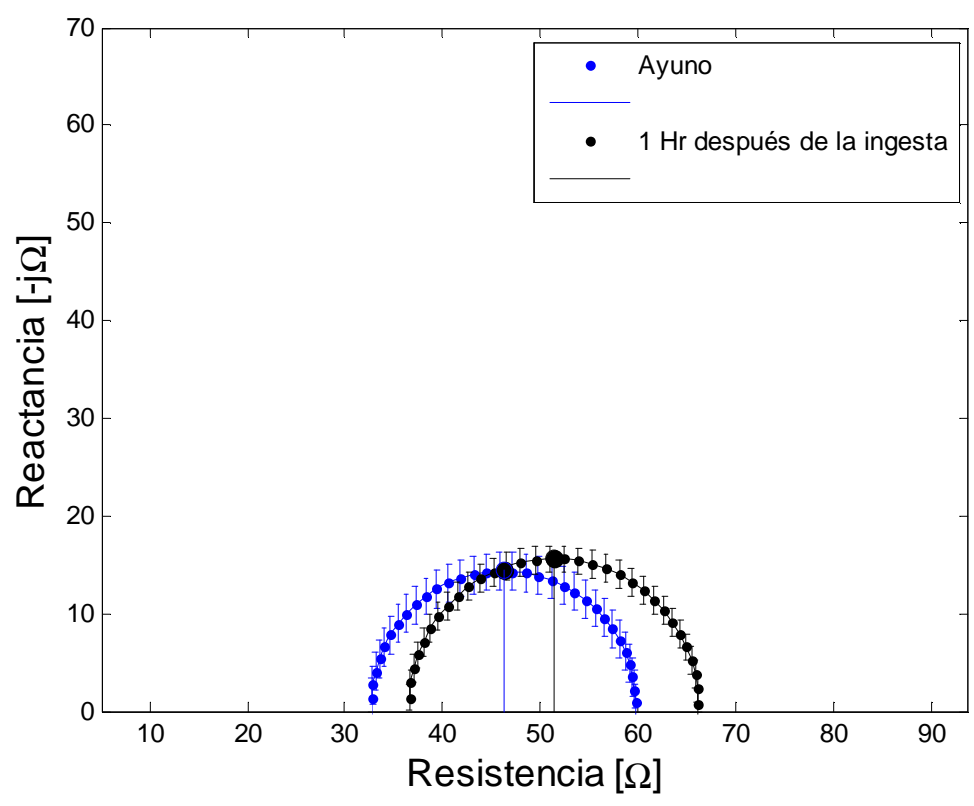

Gráfica 5. Espectro de reactancia promedio de los sujetos sanos, se observa en azul el espectro para el estado basal o en ayuno, en negro el espectro 60 minutos después de la ingesta de agua, ambas curvas corresponden al promedio \pm desviación estándar para $n=12$.

Se obtuvieron las Xh y se calculó el promedio de todos los voluntarios con su error estándar para cada condición como se muestra en la gráfica 6. Para evaluar si existen cambios en reactancia entre las condiciones de registro se realizó un ANOVA de medidas repetidas considerando resultados estadísticamente significativos con $p<0.05$. Los factores para el análisis fueron la condición y el paciente los cuales se consideraron como factor fijo y aleatorio respectivamente. Se encontró una diferencia estadísticamente significativa $(\mathbf{p}=\mathbf{0 . 0 3 5 6 7 5 )}$ entre valor de reactancia y la condición de registro.

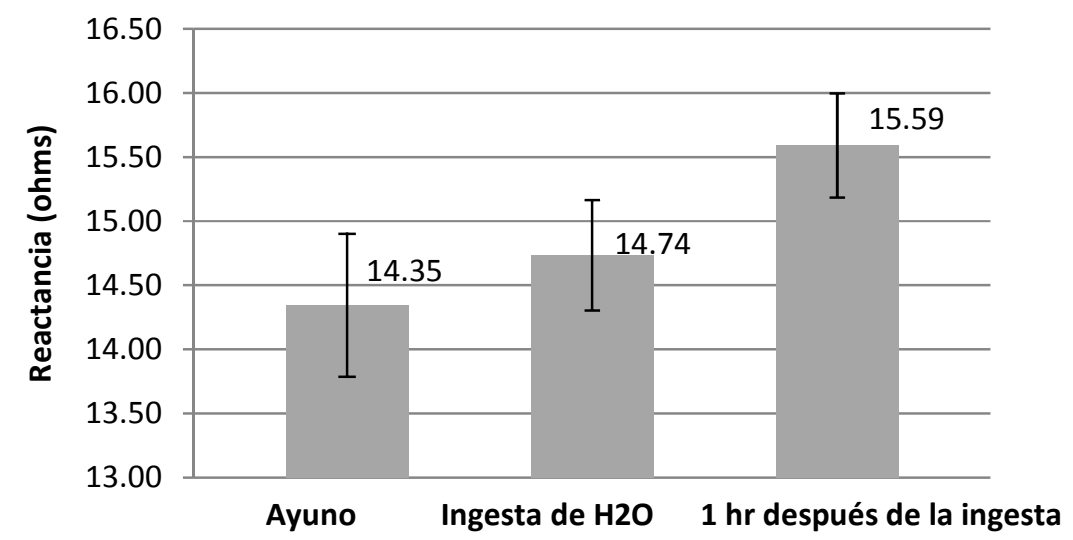

Gráfica 6. Promedio de reactancias centrales a altas frecuencias para: ayuno, ingesta de agua y 60 minutos después de la ingesta. 


\subsection{Comparación de Xh de ambas poblaciones.}

A fin de conocer si los pacientes en HDF están o no en equilibrio hídrico. Se obtuvieron los diagramas de Nyquist correspondientes a los sujetos sanos en ayuno y de los pacientes de HDF al final del tratamiento. Resulta intuitivo que los sujetos sanos estén en equilibrio hídrico al estar en ayuno ya que no existe ninguna interferencia de alimento y/o bebidas que pudieran afectar el equilibrio; mientras que en HDF al inicio del tratamiento existe un exceso de agua y toxinas mismo que se busca eliminar durante el tratamiento de tal modo que el paciente pueda alcanzar niveles normales de estos elementos. Por lo tanto si se busca el estado de equilibrio hídrico de los pacientes en HDF, es más lógico seleccionar el final de la sesión como el momento en el que se alcanzó el equilibrio hídrico. La gráfica 7 expone el diagrama de Nyquist de ambas poblaciones en su estado de equilibrio hídrico. Como se observa los sujetos de HDF poseen una mayor reactancia en comparación con los sujetos sanos.

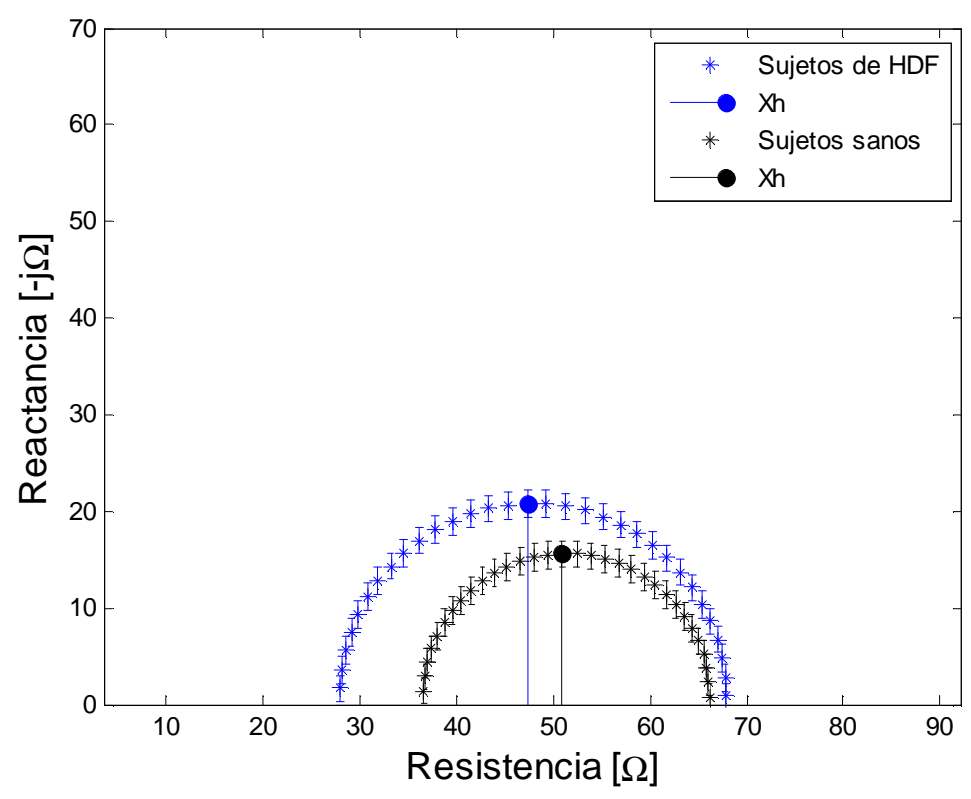

Gráfica 7. Diagrama de Nyquist obtenido a partir de la información contenida en altas frecuencias para la población de sujetos sanos y sujetos en HDF (medición \pm desviación estándar para $n=12$.

\subsection{Cambio de reactancia central y de volumen ultra filtrado}

Con la finalidad de evaluar si existe relación entre el cambio en reactancia, el cambio en VUF y el tiempo, se determinó nuevamente que el tiempo 0 corresponde al final de la sesión y al igual que en los análisis anteriores el tiempo avanza de forma negativa hasta llegar a -195 minutos tiempo en el cual se cuenta con datos completos de todos los pacientes. Se evaluó el cambio en reactancia y VUF, por lo tanto se calculó el cambio como: reactancia final menos la reactancia en el tiempo correspondiente; de tal forma que la reactancia para el tiempo 0 minutos corresponde a 0 ohms. De igual forma se realizó el cálculo para el cambio de VUF. El resultado se muestra en la gráfica 8, en ambas gráficas se obtuvo la línea de tendencia lineal y su correspondiente ecuación de la recta y el coeficiente de correlación. 


$$
\begin{aligned}
& X_{t 0}=0 \text { ohms. } \\
& X_{f}=\text { última reactancia medida } \\
& X_{t(-15 n)}=X f-X(-15 n) \quad, n=1,2,3, \ldots, 13 \\
& V_{t 0}=0 \mathrm{~L} . \\
& V_{f}=\text { último volumen de ultrafiltrado medido } \\
& V_{t(-15 n)}=V f-V(-15 n) \quad, \quad n=1,2,3, \ldots, 13
\end{aligned}
$$

a) Cambio en reactancia

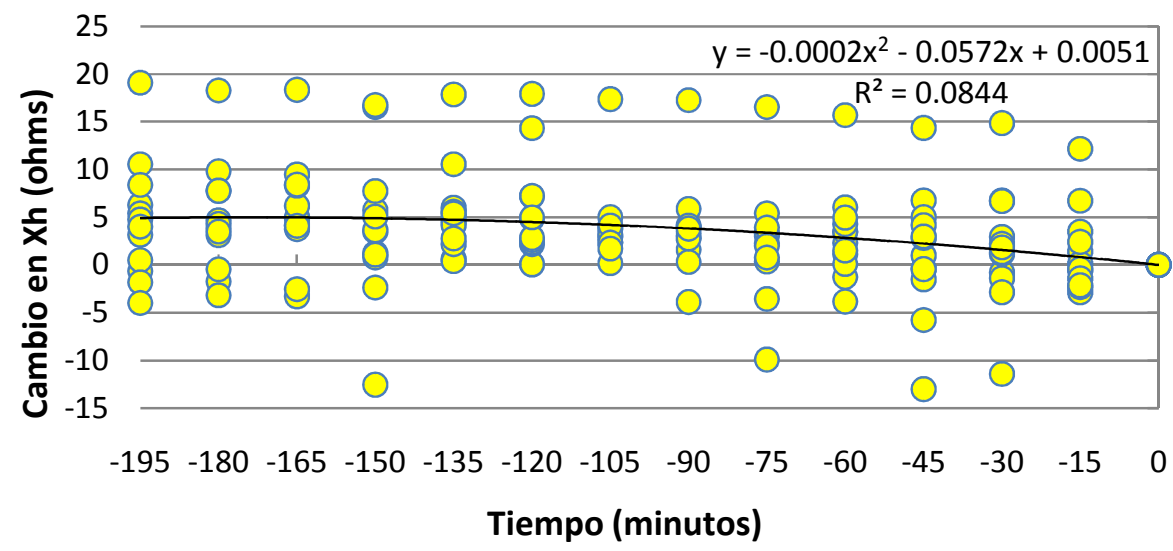

b) Cambio en el volumen de ultrafiltración

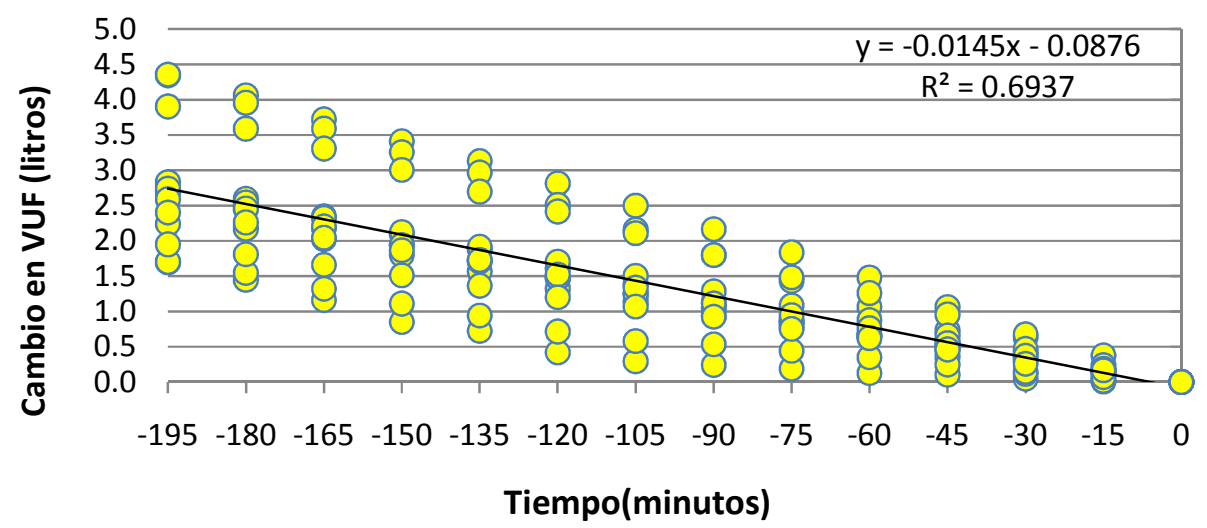

Gráfica 8. En a) se muestra el cambio en reactancia en el tiempo mientras que en b) se muestra el cambio en el volumen de ultrafiltración (VUF) en el tiempo. Ambas gráficas muestran la línea de tendencia, así como el coeficiente de determinación y la ecuación de la recta. 
Como se ve en la gráfica no existe correlación entre el tiempo transcurrido y el valor de reactancia central; mientras que para el cambio en el VUF se encontró una correlación marginalmente significativa. En la gráfica 9 se muestra la gráfica de dispersión del cambio en reactancia y VUF, al igual que las gráficas anteriores se incluye la línea de tendencia, la ecuación de la recta y el coeficiente de determinación.

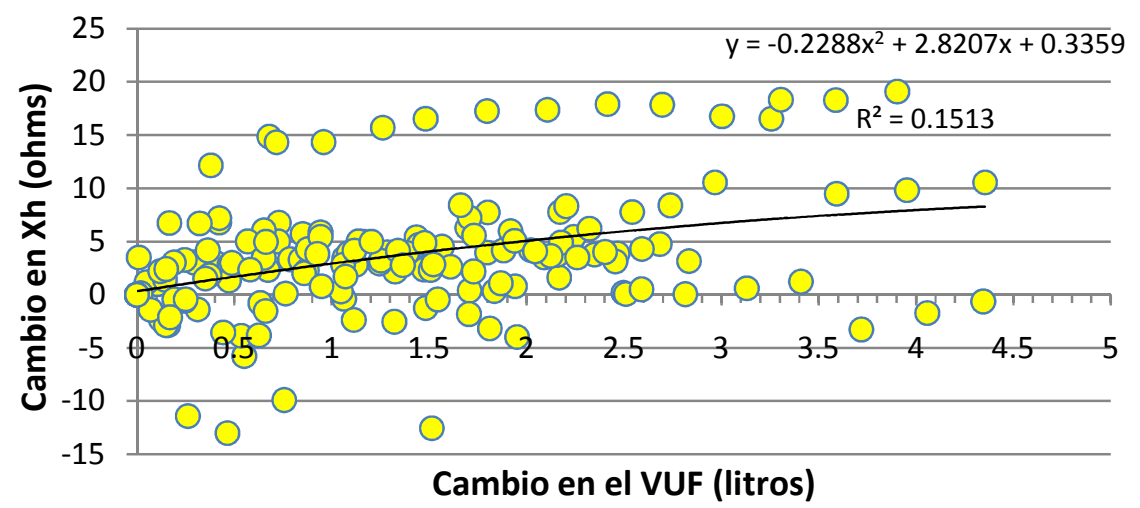

Gráfica 9. Se muestra la relación entre el cambio en reactancia y el cambio en el volumen ultrafiltrado junto con la línea de tendencia, el coeficiente de determinación y la ecuación de la recta.

Con base al coeficiente de determinación no existe correlación entre el cambio en reactancia y el volumen ultrafiltrado.

Los resultados sugieren que se debe buscar sí existe relación entre la reactancia y el tiempo para cada tratamiento y no de forma general.

\subsection{Valor predictivo de la Reactancia.}

Como se vio en la sección anterior no se encontró una buena correlación entre la reactancia y el tiempo de manera general, por lo que en esta sección se realizó un análisis de las pendientes obtenidas al graficar la reactancia en función del tiempo para cada tratamiento con la finalidad de evaluar la capacidad de detección del evento adverso. Para esto, se obtuvieron las curvas ROC para 2 criterios. El primero corresponde a la pendiente máxima de reactancia, para la cual se calcularon las pendientes punto a punto para toda la serie de tiempo seleccionando la pendiente máxima de cada tratamiento en valor absoluto. Y el segundo pertenece a la pendiente máxima del volumen ultrafiltrado.

Se calculó la curva ROC para cada tratamiento, donde se obtuvieron las áreas bajo la curva ( $A B C)$, y el error estándar del $A B C$ (E.E. $A B C$ ). En la gráfica 10 se muestran las curvas ROC para los dos criterios evaluados. Se encontró que la mayor área bajo la curva corresponde a la pendiente máxima con el $77.61 \%$. En la tabla 4 se observa el $A B C$ y el $E E A B C$ de ambos criterios. 


\begin{tabular}{ccc}
\hline Tabla 4. & Área Bajo la Curva de las pendientes \\
\hline Criterio & ABC Binomial & E.E ABC \\
P_MAX_XH_T & 0.77614 & 0.12961 \\
P_MAX_VUF_T & 0.72147 & 0.28834 \\
\hline
\end{tabular}

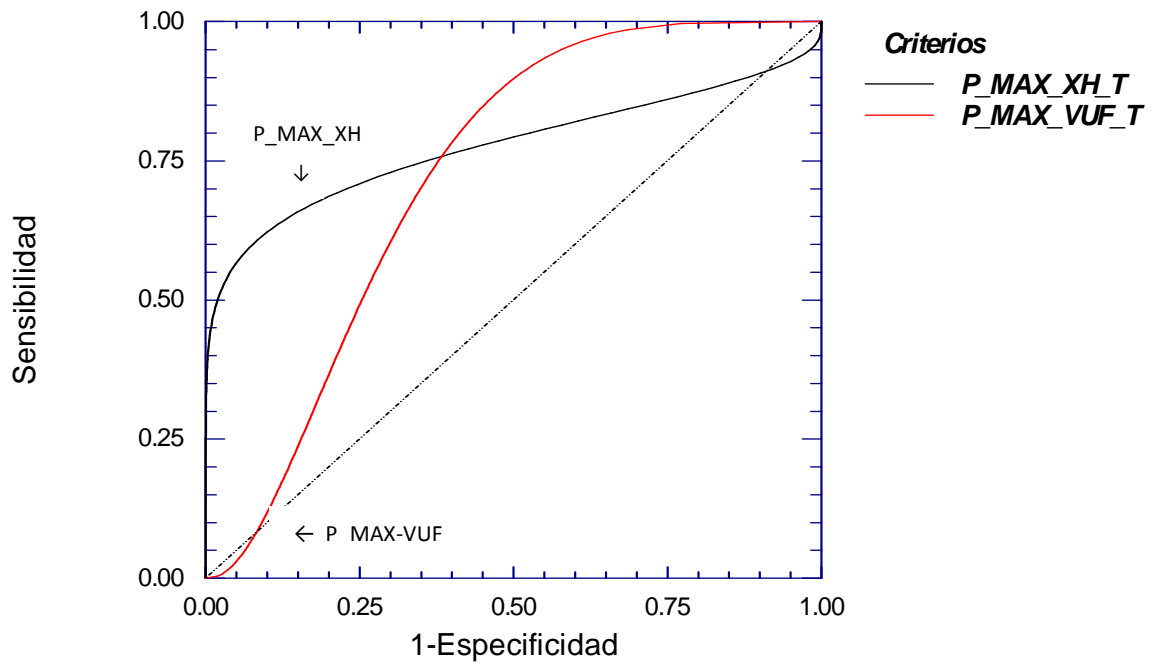

Gráfica 10. Curva ROC de los cuatro criterios evaluados, en morado se incluye la curva ROC que corresponde a 0.5 .

Para encontrar el valor de umbral de la pendiente máxima para predecir si se está o no en presencia del evento adverso, se necesita escoger el valor de corte en el cual se tenga la mayor sensibilidad y especificidad de la prueba. En la tabla 5 se muestra el valor umbral seleccionado y otros dos valores de corte que corresponden a un alta sensibilidad y especificidad respectivamente, se observa que el valor de corte seleccionado representa el mayor grado de sensibilidad y especificidad con el compromiso que esto representa. En la tabla también se muestran los resultados experimentales para la detección del evento adverso; VP corresponde a los verdaderos positivos, FP son los falsos positivo, FN falsos negativos y VN son los verdaderos positivos. Cabe mencionar que de los 12 tratamientos 7 reportaron la presencia del evento adverso.

El valor de corte seleccionado corresponde a una pendiente máxima de 0.60 , lo cual indica que por arriba de este valor se está en presencia del evento adverso.

De igual forma se calcularon los valores de corte para la pendiente máxima del cambio en el VUF.

\begin{tabular}{lllllllllll}
\hline \multicolumn{7}{c}{ Tabla 5. Valores de corte } & obtenidos de la curva ROC para la P_MAX_XH_T \\
\hline $\begin{array}{l}\text { Valor de } \\
\text { corte }\end{array}$ & VP & FP & FN & VN & Sensibilidad & Falso - & Falso + & Especificidad \\
0.27 & 7 & 5 & 0 & 0 & 0.8761 & 0.0000 & 1.000 & 0.19421 \\
$\mathbf{0 . 6 0}$ & $\mathbf{5}$ & $\mathbf{1}$ & $\mathbf{2}$ & $\mathbf{4}$ & $\mathbf{0 . 6 3 9 5}$ & $\mathbf{0 . 2 8 5 7}$ & $\mathbf{0 . 2}$ & $\mathbf{0 . 8 7 7 8 3}$ \\
0.70 & 4 & 1 & 3 & 4 & 0.5494 & 0.4285 & 0.2 & 0.96043 \\
\hline
\end{tabular}


Estimación del equilibrio hídrico tisular en hemodiálisis mediante espectroscopía de reactancia Ericka Stanford Alcántara 


\section{Discusión}

En base a los resultados es posible afirmar que la EBI de antebrazo como técnica para medir el equilibrio hídrico es sensible a los cambios de reactancia producidos al remover agua del cuerpo durante la HDF. Estudios previos permitían observar cambios en reactancia sin embargo, su reproducibilidad era muy pobre. Por esta razón, el objetivo de este trabajo consistió en mejorar la reproducibilidad intra e inter paciente. De los protocolos anteriores se identificó que la mayor fuente de variabilidad y por ende la baja reproducibilidad se debía en gran parte a las diferencias en la colocación de los electrodos. La posición de los electrodos variaba dependiendo si se pretendía realizar un análisis segmentado o completo, provocando que la distancia entre los cuatro electrodos siempre fuera diferente ; por esta razón en este trabajo se decidió realizar estudios segmentados de EBI buscando reducir al máximo las antenas formadas por los cables de adquisición; así mismo se diseñó un arreglo de electrodos de superficie con geometría constante garantizando que la superficie de contacto y la distancia entre electrodos fuera siempre la misma reduciendo la variación en las mediciones.

Se evaluó la reproducibilidad de las mediciones de EBI en el antebrazo en HDF es a partir de las gráficas $1 a$ y $1 b$, ya que ambas gráficas muestran los espectros típicos tanto de resistencia como de reactancia. En espectros ideales y en las gráficas obtenidas la pendiente máxima en la curva de resistencia corresponde con el punto más bajo o pico del lóbulo de reactancia. Otra forma de comprobar la reproducibilidad de los espectros y su relación es mediante el uso del diagrama de Nyquist (o modelo de Cole-Cole). El modelo de Cole-Cole crea un semicírculo ideal basado en los datos experimentales realizando un ajuste matemático al semicírculo ideal que más se apegue a los datos experimentales. Sí al graficar el ajuste del semicírculo y los datos experimentales estos se traslapan, querría decir que el semicírculo experimental es similar al ideal por lo tanto la variación entre mediciones es muy poca. En la gráfica 2 se muestran dos semicírculos, el ajustado y el experimental, se observó que los puntos experimentales se traslapan casi en su totalidad con el semicírculo ajustado indicando que la reproducibilidad de las mediciones es alta.

Una vez comprobada la reproducibilidad de la técnica es importante conocer que tan sensible es ante los cambios hídricos. En HDF el cambio hídrico va de la mano con el transcurso del tiempo en la sesión por lo que se obtuvieron los diagramas de Nyquist para tres momentos diferentes del tratamiento: $0 \mathrm{~min},-60 \mathrm{~min}$ y $-120 \mathrm{~min}$ (ver gráfica 3). Se encontró que el comportamiento de la reactancia coincidió con lo esperado ya que al final de la sesión o tiempo 0 min la reactancia es mayor a las reactancias en tiempos previos. La reactancia está formada por el cociente del volumen intracelular y el volumen extracelular (X=VIC/VEC), por lo que una disminución del VEC genera el aumento en reactancia. Mientras que el aumento en resistencia se explica de la siguiente forma; el líquido corporal (mezcla de iones y agua) actúa como un conductor facilitando el paso de la corriente, a medida que se extrae líquido al paciente disminuye el efecto conductor del cuerpo dificultando el paso de la corriente lo que se refleja en un aumento de la resistencia. Estos resultados establecen la base para realizar análisis posteriores ya que hasta el momento se pudo aseverar que es posible obtener mediciones reproducibles incluso intra diálisis y que la reactancia es sensible al cambio en el estado hídrico del paciente.

De lo anterior se sabe que la reactancia aumenta conforme avanza el tratamiento, sin embargo, la gráfica 4 muestra el comportamiento de la reactancia en intervalos más cortos de tiempo permitiendo visualizar los cambios punto a punto. En particular se obtuvo la ecuación de segundo 


\section{Ericka Stanford Alcántara}

orden que describe el comportamiento de la reactancia en el tiempo con un factor de correlación $\left(R^{2}=0.44\right)$. Esto nos habla de que aún existe una variación intra e interpaciente importante, sin embargo, se obtuvieron buenos resultados. En específico el modelo de la reactancia (ec. ${ }^{\text {do. }}$ orden) sugiere que existen dos momentos importantes durante el tratamiento. El primero corresponde a la fase temprana del tratamiento, en esta etapa casi no se perciben cambios en la reactancia posiblemente se deba a que el exceso de líquido acumulado en el cuerpo de los pacientes se encuentra distribuido en el plasma, LEC y LIC. Por lo que la remoción de líquidos empieza por el líquido en exceso proveniente del plasma en consecuencia el equilibrio intra-extra celular casi no se afecta en esta fase del tratamiento.

La segunda fase del tratamiento empieza cuando el volumen del plasma disminuye de tal forma que desencadena el movimiento de LEC hacia el plasma dando inicio a la extracción de LEC; es en este momento cuando la reactancia inicia su aumento. Hay que recordar que al tiempo que el VEC se ve afectado por la diminución de líquido empieza la tasa de relleno del LIC al LEC lo que eventualmente remueve el exceso de líquido de ambos compartimientos. Posiblemente este fenómeno de relleno entre compartimientos provoque que el aumento en reactancia no sea lineal.

Hasta el momento todos los resultados corresponden al análisis general de la población en HDF y el comportamiento de la reactancia, sin embargo no se conoce el comportamiento del balance hídrico de una población sana. Para esto, se sometió a la población sana a un protocoló experimental con la finalidad de medir su cambio en reactancia ante un re ajuste del equilibrio hídrico. En la gráfica 5 se muestran los diagramas de Nyquist para la condición de ayuno y la de 1 hora después de la ingesta de agua. Donde se observa que efectivamente existe un incremento en la reactancia tras la ingesta de agua. Este resultado se aprecia mejor en la gráfica 6 donde se muestra en forma de barras el promedio de $\mathrm{Xh}$ para cada condición con su respectivo error estándar. De los resultados se puede deducir los siguiente: 1) la EBI y el arreglo de electrodos permite diferenciar pequeños cambios en reactancia y 2) el aumento en reactancia aunque aún no se tiene completamente comprendido el mecanismo de absorción es ocasionado por el aumento del VIC. Suponiendo que el agua hace un recorrido desde el aparato digestivo hacia el plasma sanguíneo donde la mayor parte de líquido pasa a los riñones (a diferencia de los pacientes con IRC) mientras que el líquido que permanece en la parte vascular pasa al LEC hasta llegar al LIC provocando el aumento en reactancia.

Adicional a esto, en la sección 4.5 se compararon la población sana y la población en HDF con la intención de compara sus puntos de equilibrio hídrico. Para esto se obtuvieron sus diagramas de Cole-Cole, donde el diagrama de la población sana corresponde a la condición ayuno y el diagrama de HDF corresponde a la última medición de la población de HDF, ver gráfica 7. En este punto ambas poblaciones deberían de estar en equilibrio hídrico, no obstante observamos que la población en HDF tiene una mayor reactancia lo cual nos indica que su VEC se encuentra disminuido con respecto a los sanos con lo cual podríamos decir que de forma general se les extrajo mayor cantidad de agua que la requerida. Otro factor importante a considerar es ¿El equilibrio hídrico en la población de HDF debiera ser igual a la población sana o sí cada población tiene un equilibrio hídrico diferente? 
Dejando de lado la comparación entre poblaciones, otro objetivo del proyecto consistió en evaluar hasta qué punto los cambios en reactancia se deben a los cambios en el VUF, por ello en la sección 4.6 se muestran tres gráficas, ver gráfica $8 a, 8 b$ y 9 . Las dos primeras contiene la información de todos los pacientes del cambio en Xh y VUF para cada intervalo de tiempo con el propósito de entender su comportamiento en el tiempo. No obstante la correlación fue baja por lo que no fue posible modelar dicho comportamiento. Posiblemente al aumentar la población se pueda encontrar la ecuación que describa el cambio de Xh y VUF en el tiempo.

De la misma manera se intentó correlacionar el cambio en Xh con el cambio en VUF ya que el VUF nos indica cuanto líquido se le extrae a los pacientes. Luego entonces es de esperarse que el cambio un VUF esté relacionado con el cambio en Xh. Desafortunadamente no se encontró correlación entre la Xh y el VUF. Esto se debe posiblemente a que el VUF es la cantidad de agua removida del paciente mientras que la reactancia nos dice el equilibrio hídrico del paciente que nos indica sí la distribución de agua en el tejido es o no la correcta.

Ahora bien, para evaluar sí la reactancia es un indicador de la presencia del evento adverso y recordando que el evento adverso está relacionado con el desequilibrio hídrico, resulta lógico correlacionar la reactancia con el evento adverso. Para esto se propusieron dos criterios como posibles indicadores del evento adverso: el primero es la pendiente máxima del cambio en reactancia y segundo la pendiente máxima del cambio en el VUF. Así, mediante el uso de curvas ROC de la pendiente máxima de reactancia (P.máx. Xh) y la pendiente máxima de volumen ultrafiltrado (P.máx. VUF) se evaluó la capacidad de los criterios para discriminar el evento adverso. En la gráfica 10 se observa que las dos curvas ROC tienen un área bajo la curva (ABC) mayor a 0.5 aunque a simple vista no es posible determinar cuál de las dos tiene la mayor $A B C$. De ahí que la tabla 4 muestra que el mayor $A B C$ corresponde a la pendiente máxima del cambio en reactancia (P.máx. Xh) con $77.61 \%$ mientras que para el VUF es de $72.14 \%$, con un error estándar es de 12.9 y $28.8 \%$ respectivamente. Se eligió a la P.máx. Xh como el mejor criterio y por tanto como indicador del evento adverso. A partir de ahí seleccionó el valor umbral el cual tendrá la función de determinar si se está en presencia del evento adverso. En la tabla 5 se muestra en negritas el valor umbral seleccionado, para encontrar el valor umbral adecuado se busca que para este valor se obtenga la mayor sensibilidad y especificidad posibles bajo el entendido de que estas dos están en una relación de compromiso, es decir, al aumentar la sensibilidad se pierde especificidad y viceversa. El valor umbral para la pendiente máxima de reactancia seleccionado por cumplir con lo anteriormente mencionado es de 0.60 que corresponde a 87 y $63 \%$ de sensibilidad y especificidad respectivamente. Esto nos permite afirmar que sí la pendiente del cambio en reactancia es mayor a 0.60 se está en presencia del evento adverso. Dicho de otra forma: sí la pendiente de cambio de la reactancia es pequeña $(<0.6)$ esto indica que la extracción de líquido durante la HDF se realiza de tal forma que la disminución del VEC es compensada continuamente mediante el flujo del LIC hacia el espacio extracelular manteniendo al paciente en equilibrio hídrico, por lo contrario sí la pendiente de cambio en reactancia es mayor a 0.6 esto indica la pérdida del equilibrio hídrico generada por una tasa de extracción de LEC muy superior a la tasa de relleno desde el LIC lo que desencadena al evento adverso. 
Estimación del equilibrio hídrico tisular en hemodiálisis mediante espectroscopía de reactancia Ericka Stanford Alcántara 


\section{Conclusiones y recomendaciones}

\subsection{Conclusiones}

A través de este proyecto se validó el uso del sistema de espectroscopía de impedancia en el antebrazo mediante dos estudios: uno en pacientes sometidos a HDF y otro en voluntarios sanos; probando que es posible obtener mediciones reproducibles de reactancia en el antebrazo durante todo el tratamiento de HDF. Esto fue posible gracias a que el arreglo de electrodos diseñado otorga una geometría fija que garantiza una distancia fija entre electrodos y una superficie de contacto constante entre sujetos.

En los voluntarios sanos se encontraron diferencias estadísticamente significativas entre la reactancia central y la condición del registro. Lo que permitió considerar a la EBI de antebrazo como una técnica que permite visualizar pequeños cambios en reactancia. De igual forma en los pacientes sometidos a HDF se encontraron diferencias estadísticamente significativas en la reactancia central en diferentes momentos de la sesión. Se encontró que la reactancia aumenta conforme transcurre la sesión de HDF en consecuencia a la remoción de líquido del paciente y a la disminución del VEC.

En cuanto a la reactancia y su relación con el volumen ultrafiltrado no se encontró correlación; debido a que la reactancia estima el equilibrio hídrico que existe entre el espacio intracelular y el extracelular mientras que el VUF está relacionado a la cantidad de líquido que se extrae del paciente. Por lo que posiblemente únicamente exista correlación entre el VUF con la tasa de extracción del LEC.

El resultado de este trabajo sugiere que el cambio en la pendiente máxima de reactancia es un buen indicador de la presencia del evento adverso. Ya que evalúa la velocidad del cambio en el equilibrio hídrico y por tanto, sí el cambio resulta demasiado drástico provoca un desequilibrio hídrico importante que se traduce en la presencia del evento adverso (hipotensión, calambres, mareo, etc.). Por lo que esta técnica sugiere su posible utilización como una herramienta para monitorizar y controlar la dosis de diálisis.

A pesar de que se mejoró la reproducibilidad de las mediciones aún existe bastante variabilidad intra e interpaciente, posiblemente se deba a la distribución de masa grasa y masa magra en el antebrazo, por lo que se debe de trabajar en seleccionar una región del cuerpo en la que exista menor cantidad de masa grasa y que no se vea tan afectada por los cambios de posición. 


\subsection{Trabajo Futuro}

Basando en los resultados se siguiere modificar los conectores del equipo de espectroscopía, y utilizar otro tipo de cableado para poder disminuir el ruido. Así mismo, se sugiere experimentar con la colocación de arreglos de electrodos en diferentes regiones del cuerpo para disminuir al máximo las variaciones ocasionadas debido al cambio de posición, al movimiento del paciente y la distribución de tejido.

Se sugiere realizar estudios de EBI en poblaciones del mismo sexo y considerando su IMC para determinar si existen diferencias debidas a la composición corporal.

Como consideración adicional se propone establecer un protocolo en el cual se adquieran los registros en reposo y ante el ejercicio. Sí la ubicación del arreglo de electrodos es la adecuada la variación debida al ejercicio debería de ser mínima lo que permitiría realizar mediciones bajo cualquier condición de la sesión.

Adicionalmente se sugiere aumentar el tamaño de la muestra para optimizar el valor umbral de la pendiente en reactancia como indicador del evento adverso, así como para su validación. 


\section{Referencias}

[1] Charra B, Laurent G,Chazot C, Calemard E,. Terrat J.-C, Vanel T, Jean G, Ruffet M . "Clinical assessment of dry weight". Nephrol Dial Transplant .11. 1966

[2] Marta Arias. "La bioimpedancia como valoración del peso seco y del estado de hidratación". Dial Traspl. 31(4):137-139. 2010.

[3] Fasan Zhun,Edward F, Mary Cater, Nathan W. Levin. "Continuous Measurment of Calf Resistivity in Hemodialysis Patients using Bioimpedance Analysis". Proceedings of the $28^{\text {th }}$ IEEE EMBS Annual International Conference.USA.2006.

[4]Medrano G, Leonhardt S."Modeling the influence of body position in bioimpedance measurments". Proceedings of the $29^{\text {th }}$ IEEE EMBS Annual International Conference. France.2007.

[5] S. Soriano Cabrera. "Definición y clasificación de los estadios de la enfermedad renal crónica. Prevalencia. Claves para el diagnóstico precoz. Factores de riesgo de enfermedad renal crónica". NEFROLOGía. Vol 24. Suplemento № 6, 2004.

[6] Guyton A. "Tratado de fisiología médica" 7 ed., Ed. Philadelphia, London, Tokyo: W.B. Saunders Company, 1986.

[7] Kushner R, deVries P, Gudivaka R. "Use of bioelectrical impedance analysis measurements in the clinical management of patiente undergoing dialysis".Am J Clin Nutr.64(suppl). 1996

[8] Jaffrin $M$, Morel $H$. "Body fluid volumes measurments by impedance: A review of bioimpedance spectroscopy (Bis) and bioimpedance analysis (Bia) methods". Medical Engineering \& Physics. Vol 30. 2008

[9]Beltrán N. "Caracterización y validación clínica de espectros de impedancia de la mucosa gástrica." Tesis para obtener el grado de Doctora en ciencias de la Universidad Autónoma Metropolitana, México. 2006.

[10] Berral de la Rosa F, Rodríguez Bies E. "Impedancia bioeléctrica y su aplicación en el ámbito hospitalario". Rev Hosp Jua Mex 74.2007.

[11] Medrano G. "Continuous Monitoring of Body Fluids Using Bioimpedance Measurments." Tesis para obtener el grado de Maestro en ciencias de la Universidad de RWTH Aachen, Alemania. 2011.

[12] Gil Hernandez A. "Tratado de nutrición tomo III. Nutrición humana en el estado de salud." 2Ed. Editorial médica panamericana. Madrid. 2010.

[13] García González M, Jiménez González A, Ortiz Pedroza M, Peña Castillo M. “Potenciales bioeléctricos: origen y registro". $1^{\text {ed }}$. Universidad Autónoma METROPOLITANA. México.1988 
[14] Al-Surkhi O, Riu P, Jaffrin M. "Monitoring body fluid shifts during haemodialysis using electrical bioimpedance measurements". 1st Middle East Conference on Biomedical Engineering (MECBME).2011

[15] Piccoli A, Nescolarde L.D,Rosell J. "Análisis convencional y vectorial de bioimpedancia en la práctica clínica”. Nefrología. Vol. XXII. Número 3. 2002

[16] Matthie J, Zarowitz B, De Lorenzo A, Andreoli A, Katzarski K, Pan G, Withers P."Analytic assessment of the various methods used to estimate body water". J Appl Physiol 84. 1998.

[17] Lukaski H. "Biological indexes considered on the derivation of the bioelectrical impedance analysis". Am J Clin Nutr.64. 1966

[18] Kushner R, de Vries P, Gudii'aka R. "Use of bioelectrical impedance analysis measurements in the clinical management of patients undergoing dialysis". Am J Clin Nutr.64. 1966

[19] Beltrán G, "Correlación del cambio del volumen mediante espectrpscopía de reactancia en pacientes bajo tratamiento hemodialítico". Proyecto Terminal para obtener el grado de Ingeniero Biomédico. Universidad Autónoma Metropolitana. 2011.

[20] Tedner B, Lins L, Asaba H, Wehle B ."Evaluation of impedance techniques for fluid-volume monitoring during hemodialysis". International Journal of Clinical Monitoring and Computing 2. 1985

[21] Amaro P, García J, Jimeno R. "Evolución del peso seco en pacientes ancianos en hemodiálisis y posible influencia de la actividad física en el mismo". Gerokomos: Revista de la Sociedad Española de Enfermería Geríatrica y Gerontológica, ISSN 1134-928. Vol.21,1.2010.

[22] Othman S, Sacristan Rock E. "Complex impedance spectrometer using parallel demodulation and digital conversion.US patent No. 6,970,783, 2005.

[23] I. Czerkiewicz "Trastornos de la osmolaridad Interpretación y diagnóstico etiológico". Acta Bioquím Clín Latinoam 2004; 38 (2): 203-20. 